\title{
Binary Interaction between Typhoons Fengshen (2002) and Fungwong (2002) Based on the Potential Vorticity Diagnosis
}

\author{
Chung-Chuan Yang, Chun-Chieh Wu, Kun-Hsuan Chou, and Chia-Ying Lee \\ Department of Atmospheric Sciences, National Taiwan University, Taipei, Taiwan
}

(Manuscript received 17 December 2007, in final form 16 April 2008)

\begin{abstract}
A cyclonic loop was observed in the track of Typhoon Fungwong (2002) when it was about $765 \mathrm{n}$ mi from Supertyphoon Fengshen (2002). It is shown that Fungwong's special path is associated with the circulation of Fengshen, and such an association is regarded as an indication of binary interaction. In this paper, the binary interaction between Fengshen and Fungwong is studied based on the potential vorticity diagnosis. The impacts of large-scale flow fields on their motions are also investigated. Furthermore, the sensitivity of the storm characteristics to the binary interaction is demonstrated by the mesoscale numerical model simulations with different sizes and intensities for the initial bogused storms. Results of the study show that before Fungwong and Fengshen interacted with each other, their motions were governed by the large-scale environmental flow, that is, mainly associated with the subtropical high. During this binary interaction, Fungwong's looping is partly attributed to Fengshen's steering flow. This pattern shows up first as a case of one-way interaction in the early period, and then develops into a mutual interaction during the later stages. The numerical experiments show the sensitivity of the storm size and intensity to the binary interaction, implicating that a good representation of the initial storm vortex is important for the prediction of binary storms. Further analyses also indicate the influence of the monsoon trough and subtropical high systems on the binary interaction. These results provide some new insights into the motions of nearby typhoons embedded in the monsoon circulation.
\end{abstract}

\section{Introduction}

The concept of binary interaction has been introduced through many experiments (Fujiwhara 1921, 1923, 1931), observations (Brand 1970; Dong and Neumann 1983; Lander and Holland 1993; Carr et al. 1997; Carr and Elsberry 1998), and modeling studies (Chang 1983, 1984; DeMaria and Chan 1984; Ritchie and Holland 1993; Holland and Dietachmayer 1993; Wang and Holland 1995). Taken together, these works have well described the binary interaction between two tropical cyclones (TCs), including the mutual orbiting, approaching, and merging, along with the escaping processes (Lander and Holland 1993). The binary interaction is not only an interesting and fundamental issue in

Corresponding author address: Dr. Chun-Chieh Wu, Dept. of Atmospheric Sciences, National Taiwan University, No. 1, Sec. 4, Roosevelt Rd., Taipei 106, Taiwan.

E-mail: cwu@typhoon.as.ntu.edu.tw fluid mechanics and vortex dynamics but also a practical concern in improving the forecast accuracy of multiple TCs in close vicinity to each other.

Early laboratory experiments by Fujiwhara (1921, 1931) studied the interaction of binary vortex systems and found that in addition to the mutual rotational effect, there exists a tendency for an attraction and rotation between vortices. A meteorological analogy to these laboratory vortices occurs when two TCs become close enough for mutual interaction. Observational evidence (Brand 1970) suggests a correlation between the separation distance and the angular rotation rate of two TCs in a binary interaction, which depends on the differences in storm size, intensity, and the variations in the currents. Specifically, Brand showed that when the distance between two TCs is under $750 \mathrm{n} \mathrm{mi}$, they begin to circle around each other. When the distance is less than $400 \mathrm{n} \mathrm{mi}$, they are slightly attracted to each other.

However, Lander and Holland (1993) showed that instead of following the classic Fujiwhara model, a se- 
ries of interactions including capturing, stable cyclonic orbiting, and cyclone merging and escaping, take place during the interaction of cyclones. Carr et al. (1997) and Carr and Elsberry (1998) had proposed detailed conceptual modes to categorize the binary interaction processes, namely, 1) the direct TC interaction with oneway influence, mutual interaction, or the merger of two $\mathrm{TCs} ; 2)$ the semidirect TC interaction involving another $\mathrm{TC}$ and an adjacent subtropical anticyclone; and 3) the indirect TC interaction involving the anticyclone between the TCs. The interaction between two TCs with different core vorticities and different sizes is studied by Prieto et al. (2003) with the aid of a nondivergent barotropic model, on both the $f$ plane and the sphere, and with the use of the Dritschel and Waugh (1992) scheme, in which the vortex interaction is subdivided into five types: elastic interaction, partial straining out, complete straining out, partial merger, and complete merger.

Despite the aforementioned research efforts, there are issues that remain to be addressed, including 1) factors that affect the binary interaction, such as the storm size, intensity, location, the separation distance, and the flow patterns around TC systems, and 2) quantitative assessment of binary interaction.

To provide a quantitative assessment of binary interaction, Wu et al. (2003) conducted an analysis from a potential vorticity (PV) perspective, namely, a newly proposed centroid-relative track. This centroid-relative track, with the position weighting based on the steering flow induced by the PV anomaly associated with another storm, is plotted to highlight the binary interaction process. Moreover, the adoption of the piecewise PV inversion (Davis 1992; Wu and Emanuel 1995a,b) provides a means to quantitatively evaluate how the binary TCs interact with each other. In addition, Wu et al. (2004) applied the same PV diagnosis to study the factors contributing to the decrease in the translation speed of Typhoon Sinlaku (2002). The PV diagnosis provides a useful approach to determining the key factors affecting the typhoon track quantitatively, and provides a better insight into improving future observing and modeling strategies and, thus, better typhoon forecasts.

As a follow-up work of $\mathrm{Wu}$ et al. $(2003,2004)$, this study on the binary interaction between Typhoons Fengshen (2002) and Fungwong (2002) is conducted, and the impacts of the large-scale flow fields on their motions are examined. Furthermore, the binary interaction between Fengshen and Fungwong is investigated via mesoscale numerical model simulations with different initial conditions and different bogused storm vortices. The sensitivity of the vortex characteristics to the binary interaction is demonstrated, and is used not only to explore the factors controlling the interaction of binary vortices, but also to further examine the impact of the uncertainty of the initial vortex conditions on the interaction of nearby TCs. The major objectives of this paper include the following:

- evaluation of the binary interaction based on the PV diagnosis,

- investigation of the impact of the large-scale flow fields on the motions of both Fengshen and Fungwong,

- exploration of the factors that control the interaction of binary vortices by numerical model simulations, and

- examination of the impacts of the uncertainty of the initial vortex conditions on the interaction of the nearby TCs.

The data and model used in this study are presented in section 2, while the analysis method and experiment design are described in section 3 . Results are detailed in sections 4 and 5 , followed by the conclusions in the final section.

\section{Data and model description}

\section{a. Data}

The datasets used for this study are the analysis fields of the Tropical Ocean and Global Atmosphere experiment from the European Centre for Medium-Range Weather Forecasts (EC_TOGA). To see the overall picture of the whole interaction process between Fengshen and Fungwong, the PV analysis of the 12-hourly EC_TOGA data from 0000 UTC 20 July to 0000 UTC 27 July 2002, covering the lifetime of Fungwong, is conducted based on the PV diagnosis method (Wu et al. $2003,2004)$, within the domain of $5^{\circ}-50^{\circ} \mathrm{N}$ and $100^{\circ} \mathrm{E}-$ $180^{\circ}$.

\section{b. Model description}

A version of the fifth-generation Pennsylvania State University-National Center for Atmospheric Research Mesoscale Model (MM5; Anthes and Warner 1978; Anthes et al. 1987), which is a limited-area, nonhydrostatic, terrain-following sigma-coordinate model designed to simulate or predict mesoscale atmospheric circulation, is used in this study. A suite of model physical parameterization schemes is available for subgridscale convection, a grid-resolvable scale, microphysical parameterization, planetary boundary layer physics, and radiation. 


\section{Analysis method and experimental design}

a. Analysis method

The diagnosis method of $\mathrm{Wu}$ et al. $(2003,2004)$ is adopted in this study, including the piecewise PV inversion, the deep-layer mean steering flow, AT (the normalized steering effect associated with each PV perturbation in the along-track direction), and the newly defined centroid-relative tracks.

The merit of the PV inversion is that given a distribution of the PV, a prescribed balance condition, and the boundary conditions, the balanced mass and wind fields can be recovered. The special advantage of the PV inversion method comes from the so-called piecewise PV inversion; that is, when the flow field is divided into the mean and perturbation components, the balance fields associated with each individual PV perturbation could be obtained from the perturbation PV equations (Davis 1992). Such a method can describe how the different PV features and the environment perturbations affect the TC track (Wu and Emanuel 1995a,b; Shapiro 1996, 1999; Wu and Kurihara 1996; Wu et al. 2003, 2004; Wu and Wang 2000, 2001).

To understand how Fengshen and other environmental flows affected the track of Fungwong, the axisymmetric average relative to the center of Fungwong is taken as the mean part, and the approach by Wu et al. (2003, 2004) is followed to perform the piecewise PV inversion. To obtain the average streamfunction $\bar{\Psi}$ the azimuthal average of the wind field is constructed so that the associated average geopotential height $(\hat{\Phi})$ is derived from the nonlinear balanced equation, and the averaged PV field $(\hat{q})$ is calculated by the following relation:

$$
\begin{aligned}
\hat{q}= & \frac{g k \pi}{p}\left[\left(f+\nabla^{2} \bar{\Psi}\right) \frac{\partial^{2} \hat{\Phi}}{\partial \pi^{2}}-\frac{1}{a^{2} \cos ^{2} \phi} \frac{\partial^{2} \bar{\Psi}}{\partial \lambda \partial \pi} \frac{\partial^{2} \hat{\Phi}}{\partial \lambda \partial \pi}\right. \\
& \left.-\frac{1}{a^{2}} \frac{\partial^{2} \bar{\Psi}}{\partial \phi \partial \pi} \frac{\partial^{2} \hat{\Phi}}{\partial \phi \partial \pi}\right]
\end{aligned}
$$

where $q$ represents $\mathrm{PV}, \Phi$ represents geopotential height, $\Psi$ represents the streamfunction, $a$ is the earth's radius, $f$ is the Coriolis parameter, $\phi$ is the longitude, $\lambda$ is the latitude, and $\pi$ is the pressure coordinate, $\pi=$ $C_{p}\left(P / P_{0}\right)^{\kappa}$, with $\kappa=R_{d} / C_{p}$. By taking the perturbation field as $\Psi^{\prime}=\Psi-\bar{\Psi}, \Phi^{\prime}=\Phi-\hat{\Phi}$, and $q^{\prime}=q-\hat{q}$, the piecewise $\mathrm{PV}$ inversion is performed to calculate the balanced flow and mass fields associated with each PV perturbation.

The steering flow of each storm is defined as the deep-layer-mean wind vector average over the inner $3^{\circ}$ latitude around the storm's center (based on the location of the maximum $\mathrm{PV}$ at $850 \mathrm{hPa}$ ):

$$
\mathbf{V}_{\mathrm{SDLM}}=\frac{\int_{925 \mathrm{hPa}}^{300 \mathrm{hPa}} \mathbf{V}_{S}(p) d p}{\int_{925 \mathrm{hPa}}^{300 \mathrm{hPa}} d p},
$$

where

$$
\mathbf{V}_{S}(p)=\frac{\int_{0}^{2 \pi} \int_{0}^{3^{\circ}} \mathbf{V} r d r d \theta}{\int_{0}^{2 \pi} \int_{0}^{3^{\circ}} r d r d \theta}
$$

To quantitatively measure the influence of the steering flow associated with some particular PV perturbation, the component of the steering flow associated with $q_{s}^{\prime}\left[\right.$ i.e., $\left.\mathbf{V}_{\text {SDLM }}\left(q_{s}^{\prime}\right)\right]$ in the direction parallel to the steering flow vector associated with total PV perturbations $\left(q^{\prime}\right)$ is defined as

$$
\operatorname{AT}\left(q_{S}^{\prime}\right)=\frac{\left|\mathbf{V}_{\text {SDLM }}\left(q_{S}^{\prime}\right) \cdot \mathbf{V}_{\text {SDLM }}\left(q^{\prime}\right)\right|}{\left|\mathbf{V}_{\text {SDLM }}\left(q^{\prime}\right)\right|^{2}},
$$

where $\mathbf{V}_{\text {SDLM }}\left(q_{S}^{\prime}\right)$ and $\mathbf{V}_{\text {SDLM }}\left(q^{\prime}\right)$ have been defined in Eq. (2) and the $q_{s}^{\prime}$ indicates the particular PV perturbation. Note that AT is a normalized quantity and, by definition, $\operatorname{AT}\left(q^{\prime}\right)=\operatorname{AT}\left(q_{S}^{\prime}\right)+\operatorname{AT}\left(q_{\text {nos }}^{\prime}\right)$, where $q_{\text {nos }}^{\prime}$ represents the rest of the PV perturbation other than $q_{s}^{\prime}$, and $q^{\prime}=q_{s}^{\prime}+q_{\text {nos. }}^{\prime}$.

Following Wu et al. (2003), the centroid is defined as the location weighted according to the strength of the steering flow induced by the PV perturbation associated with each storm:

$$
\begin{aligned}
\mathbf{r}_{c}= & \frac{\left|\mathbf{V}_{\mathrm{SDLM}}\left(q_{\mathrm{FS}}^{\prime}\right)\right|}{\left|\mathbf{V}_{\mathrm{SDLM}}\left(q_{\mathrm{FS}}^{\prime}\right)\right|+\left|\mathbf{V}_{\mathrm{SDLM}}\left(q_{\mathrm{FW}}^{\prime}\right)\right|} \mathbf{r}_{F S} \\
& +\frac{\left|\mathbf{V}_{\mathrm{SDLM}}\left(q_{\mathrm{FW}}^{\prime}\right)\right|}{\left|\mathbf{V}_{\mathrm{SDLM}}\left(q_{\mathrm{FS}}^{\prime}\right)\right|+\left|\mathbf{V}_{\mathrm{SDLM}}\left(q_{\mathrm{FW}}^{\prime}\right)\right|} \mathbf{r}_{\mathrm{FW}},
\end{aligned}
$$

where the suffixes FS and FW represent the PV perturbations associated with Fengshen and Fungwong, respectively, and $\mathbf{r}$ represents the position vector of the storm. It is expected that the centroid should be located closer to the more dominating TC in the binary interaction process.

\section{b. Experimental design}

A series of sensitivity experiments are conducted to explore the factors controlling the interactions of binary vortices. The model configuration includes two nested grids, $109 \times 139$ grids in the outer nest and $151 \times 208$ grids in the inner nest, with horizontal resolutions of 60 and $20 \mathrm{~km}$, respectively, and 23 sigma levels in the vertical. The initial and boundary conditions are taken from EC_TOGA analyses. Each simulation is integrated for $72 \mathrm{~h}$, starting from 0000 UTC 23 

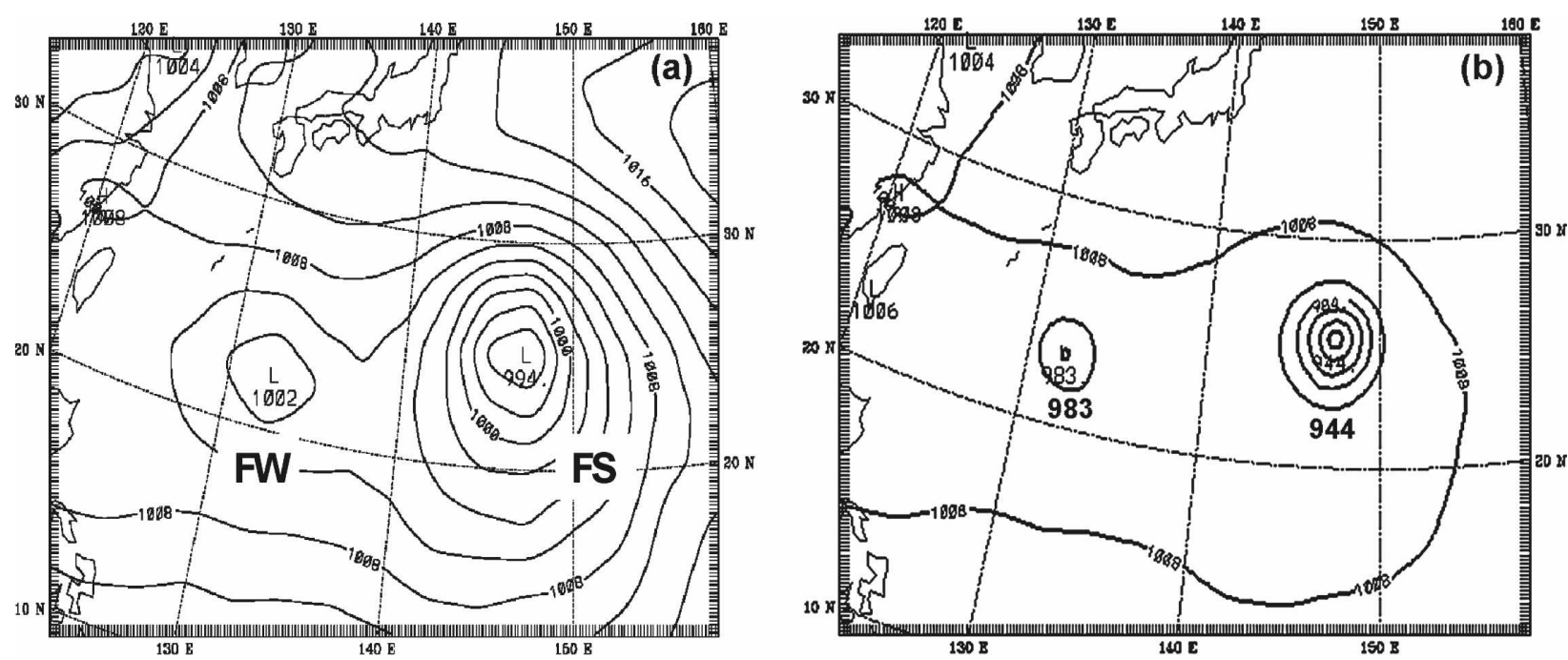

FIG. 1. Sea level pressure at 0000 UTC 23 Jul 2002. (a) Analysis of EC_TOGA without the bogused initialization (contour interval of $2 \mathrm{hPa}$ ). (b) As in (a) but with the bogused initialization (contour interval of $12 \mathrm{hPa}$ ). The central sea level pressures of Fengshen and Fungwong are labeled.

July 2002 with two bogused vortices based on the vortex initialization method in Wu et al. (2002). Without this initialization method, the initial analyses of the minimum sea level pressures of Fungwong and Fengshen are 1002 and $994 \mathrm{hPa}$, respectively, which are much weaker than the best-track analyses of 985 and $940 \mathrm{hPa}$ from the Japan Meteorological Agency (JMA) at 0000 UTC 23 July (Fig. 1a). Figure 1b shows the initially simulated intensities of the two typhoons after performing the model initialization. After implanting the bogused vortices of the two storms, the corresponded intensities reach 983 and $944 \mathrm{hPa}$, respectively, which are in very good agreement with the analyses from JMA.

The list of experiments is shown in Table 1, where the sensitivity experiments are associated with different TC sizes, intensities, relative locations, and environmental flows. In the control experiment, denoted as CTRL, the initial sizes of the bogused vortices for Fengshen and Fungwong are 360 and $300 \mathrm{~km}$, respectively. For the sensitivity experiments, some parameters are changed as described below.

In experiment B_FS, the initial size of Fengshen is increased to highlight the influence of the storm size on the binary interaction; in experiment S_FW, the initial intensity of Fungwong is increased to $944 \mathrm{hPa}$. In this case, the experiment starts with two vortices of equal intensity; similar to S_FW, in W_FS, the simulation starts with two vortices with the same intensity but of $983 \mathrm{hPa}$. In other words, in W_FS, Fengshen's intensity is reduced to the same as that of Fungwong. The above two experiments, W_FS and S_FW, are designed to in- vestigate the influence of the storm intensity on binary interaction. In experiment FW (FS), the storm vortex of Fengshen (Fungwong) is removed to examine the track of each storm without the binary interaction and to isolate the influence of favorable environmental flow, such as the monsoon trough (Lander 1994, 1996), on the storm track. The final experiment, denoted as REVS, is conducted by switching the two storm vortices at the initial time.

\section{Results of global analysis data}

\section{a. Synopsis}

\section{1) Supertyphoon Fengshen}

The long-lived and intense Supertyphoon Fengshen traveled from its birthplace near the eastern Marshall Islands to its final landfall as a weak depression system

TABLE 1. List of experiments. The open (filled) cyclone symbol indicates the weaker vortex with minimum sea level pressure of 983 (944) hPa. The enlarged cyclone symbol indicates the vortex with bigger size.

\begin{tabular}{lccl}
\hline \hline Expt & Fungwong & Fengshen & \multicolumn{1}{c}{ Remarks } \\
\hline CTRL & 9 & 6 & Control run \\
B_FS & 9 & 6 & $\begin{array}{l}\text { Fengshen with bigger vortex } \\
\text { size }\end{array}$ \\
S_FW & 6 & 6 & Fungwong with stronger vortex \\
W_FS & 9 & 9 & Fengshen with weaker vortex \\
FW & 9 & - & Fengshen vortex removed \\
FS & - & 9 & Fungwong vortex removed \\
REVS & 9 & 6 & Switch of vortices \\
\hline
\end{tabular}




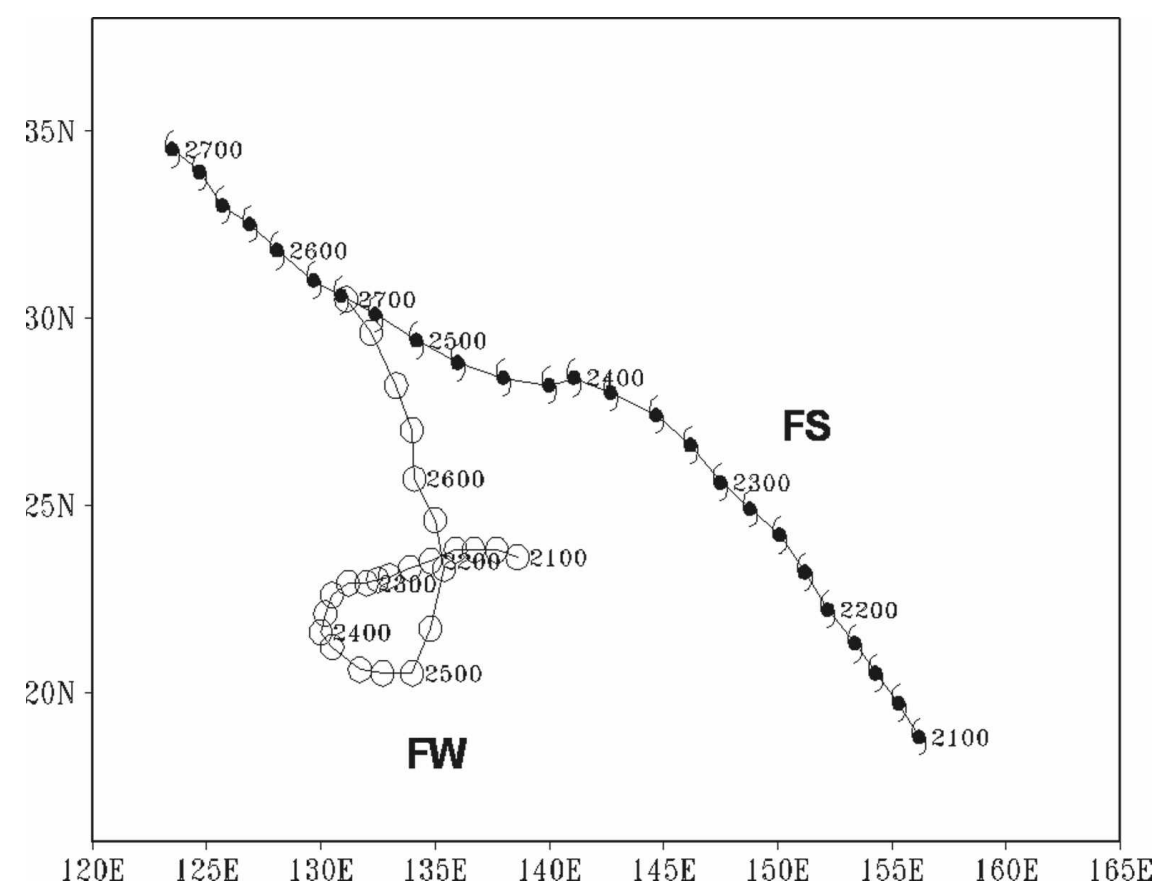

FIG. 2. JTWC's best tracks of Fengshen and Fungwong from 0000 UTC 21 Jul to 0000 UTC 27 Jul 2002.

on China's Shandong Peninsula (G. Padgett 2002, personal communication). Fengshen was recorded as a moderate-strength typhoon at 0000 UTC 15 July 2002 by JMA. As the subtropical ridge to the north strengthened, it slowly turned to the west. By 0000 UTC 18 July, Fengshen's maximum surface wind had increased to $130 \mathrm{kt}$ and had become the fifth supertyphoon in 2002. On 21 July, Fengshen moved northwestward steadily and intensified to its peak value of $145 \mathrm{kt}$ at $1200 \mathrm{UTC}$ 21 July under the influence of environmental flow, such as the upper-level flow, and the poleward and equatorward outflows.

Fengshen continued northwestward on 22 July and then weakened early on 23 July when its strength dropped to $120 \mathrm{kt}$. Fengshen's west-northwesterly motion had gradually increased to $19 \mathrm{kt}$ at 1800 UTC 23 July due to a strengthening midlevel ridge over central Japan and perhaps due to the interaction with Typhoon Fungwong, which was located approximately $765 \mathrm{n} \mathrm{mi}$ west-southwest of Fengshen at the time (Fig. 2). Fengshen was reduced to tropical storm status at 0600 UTC 25 July and made landfall on the southern Shandong Peninsula around 1700 UTC 27 July as a tropical depression with the maximum surface wind of $30 \mathrm{kt}$.

\section{2) TypHOOn FungwONG}

Typhoon Fungwong, with its track strongly affected by Supertyphoon Fengshen, was a case in point to show the Fujuwhara effect. Fungwong was located at about $70 \mathrm{n} \mathrm{mi}$ west-southwest of Iwo Jima as a tropical depression at 1200 UTC 20 July, and then reached tropical storm status at 0000 UTC 21 July with JMA assigning the name, Fungwong. Fungwong steadily moved westward on 21 July, guided by a low-level ridge to the north, and then began to move southwestward as Typhoon Fengshen approached from the east. Fungwong was tracked south-southwestward by 1800 UTC 23 July and apparently began to experience the influence of Fengshen, located $765 \mathrm{n} \mathrm{mi}$ to the east-northeast. At 0000 UTC 24 July, the track of Fungwong curved rather sharply to the south and then to the east-southeast because of the influence of Fengshen. Fungwong was downgraded to tropical depression status at 0000 UTC 27 July, with the final position located about $455 \mathrm{n} \mathrm{mi}$ from the weakening Fengshen (Fig. 2).

\section{b. PV diagnosis}

To assess the mechanism of the interaction between Fengshen and Fungwong, the PV $(q)$ at $500 \mathrm{hPa}$ and the deep-layer-mean wind fields $(925-300 \mathrm{hPa})$ are shown in Fig. 3.

At 0000 UTC 21 July (Fig. 3a), Fengshen and Fungwong are both located in a high-PV region, with maximum PV of 1.0 PVU (1 PVU $\left.=10^{-6} \mathrm{~m}^{2} \mathrm{~s}^{-1} \mathrm{~K} \mathrm{~kg}^{-1}\right)$ and 0.7 PVU, respectively. Fengshen strengthens to 1.5 PVU at 0000 UTC 24 July and maintains its intensity 

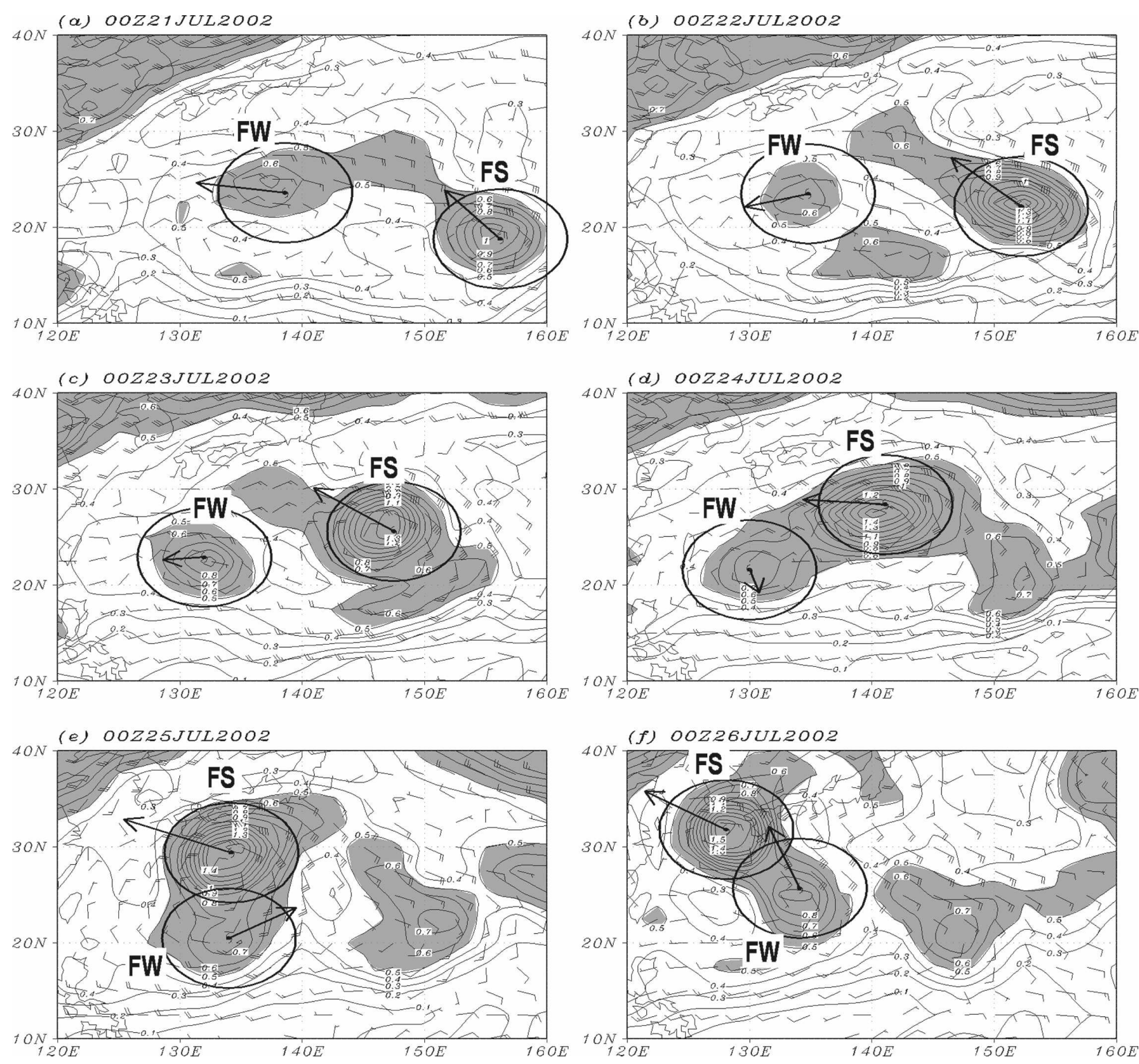

FIG. 3. The PV (contour interval of 0.1 PVU; PV larger than 0.5 PVU is shaded) at $500 \mathrm{hPa}$ and the 925-300-hPa deep-layer-mean wind (one full wind bar $=5 \mathrm{~m} \mathrm{~s}^{-1}$ ) at 0000 UTC (a) 21, (b) 22, (c) 23, (d) 24, (e) 25, and (f) 26 Jul 2002. The typhoon symbols represent the positions of Fengshen and Fungwong. The instantaneous movement of each storm is indicated by the boldface arrow, whose length represents the actual translation velocity; the boldface circle shows the scale of $5 \mathrm{~m} \mathrm{~s}^{-1}$.

for the next 2 days while the intensity of Fungwong stays between 0.7 and 0.9 PVU. As shown in Figs. 2 and 3 , Fengshen heads northwest from 21 to 26 July, except at 0000 UTC 24 July when it obviously moves westward, whereas Fungwong moves westward from 21 to $23 \mathrm{July}$, and then experiences a cyclonic looping from 24 to 26 July, probably under the strong influence of Fengshen. On the other hand, Fengshen, unlike Fungwong, only changes slightly in its track instead of performing a severe turn during this period.
The total PV perturbation component $\left(q^{\prime}\right)$ and its deep-layer-mean wind fields are shown in Fig. 4. Fungwong's movement closely follows the deep-layer-mean wind field. The relation between the instantaneous motion of Fungwong and the balanced deep-layermean flow associated with the PV perturbation of Fengshen is presented in Fig. 5, which demonstrates how Fengshen affects Fungwong's motion. Clearly, Fengshen has a trivial effect on Fungwong on 21 July, while the balanced steering flow associated with Feng- 

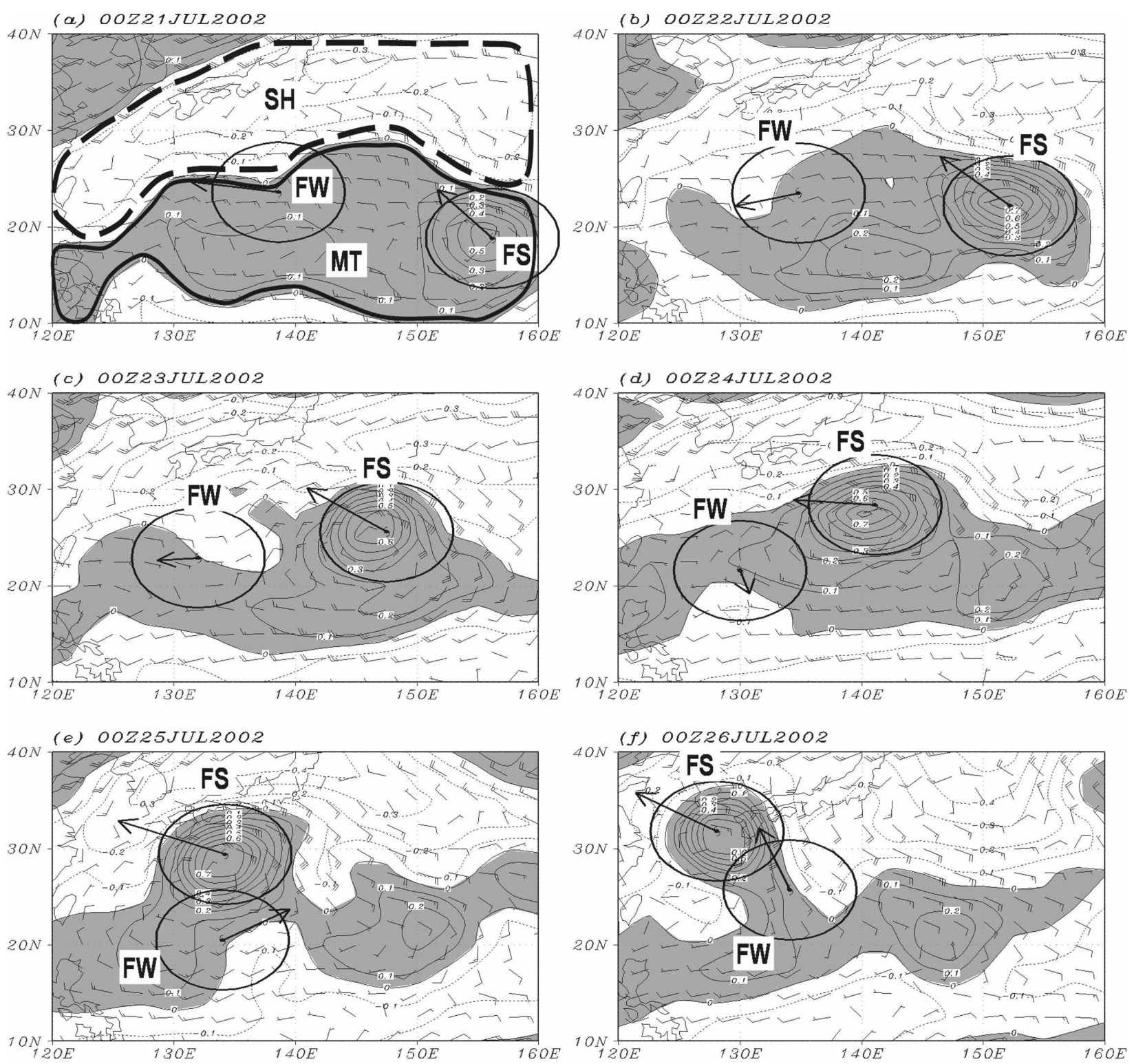

FIG. 4. Total PV perturbation (with Fungwong chosen as the mean flow), $q^{\prime}$, (contour interval of 0.1 PVU; the positive PV perturbation is shaded) at $500 \mathrm{hPa}$, and the $925-300-\mathrm{hPa}$ deep-layer-mean wind (one full wind barb $=5 \mathrm{~m} \mathrm{~s}^{-1}$ ) at $0000 \mathrm{UTC}$ (a) 21 , (b) 22, (c) 23, (d) 24, (e) 25, and (f) 26 Jul 2002. The definitions of the boldface arrow and circle are as in Fig. 3. Here, FS and FW indicate the locations of Fengshen and Fungwong, respectively. The solid and dashed curves represent the areas of PV perturbations associated with MT and SH, respectively.

shen more strongly affects Fungwong's motion from 24 to 25 July.

\section{1) Movement of Fungwong}

To evaluate the steering flow associated with various PV perturbations, following Wu et al. $(2003,2004)$, the time series of the deep-layer-mean steering flow associated with the total $\left[\mathbf{V}_{\text {SDLM }}\left(q^{\prime}\right)\right]$ and each PV perturbation $\left[\mathbf{V}_{\text {SDLM }}\left(q_{\mathrm{FS}}^{\prime}\right), \mathbf{V}_{\text {SDLM }}\left(q_{\mathrm{noFS}}^{\prime}\right), \mathbf{V}_{\mathrm{SDLM}}\left(q_{\mathrm{SH}}^{\prime}\right)\right.$, $\left.\mathbf{V}_{\text {SDLM }}\left(q_{\text {noSH }}^{\prime}\right), \mathbf{V}_{\text {SDLM }}\left(q_{\mathrm{MT}}^{\prime}\right), \mathbf{V}_{\text {SDLM }}\left(q_{\text {noMT }}^{\prime}\right)\right]$ are com- pared with the actual movement of Fungwong [estimated from the 12-h best-track positions, i.e., $\mathbf{V}_{\mathrm{BT}}=$ $\left.\left(\mathbf{X}_{t+6 \mathrm{~h}}-\mathbf{X}_{t-6 \mathrm{~h}}\right) / 12 \mathrm{~h}\right]$. As illustrated in Fig. 4a, the acronyms FS, SH, and MT indicate the perturbations associated with Fengshen, the subtropical high, and the monsoon trough, respectively. Note that the monsoon trough is indicated in Fig. 4a as the zonally elongated positive PV perturbation belt in which Fengshen is embedded. Meanwhile, noFS (noSH, noMT) indicates the perturbation other than Fengshen (the subtropical 

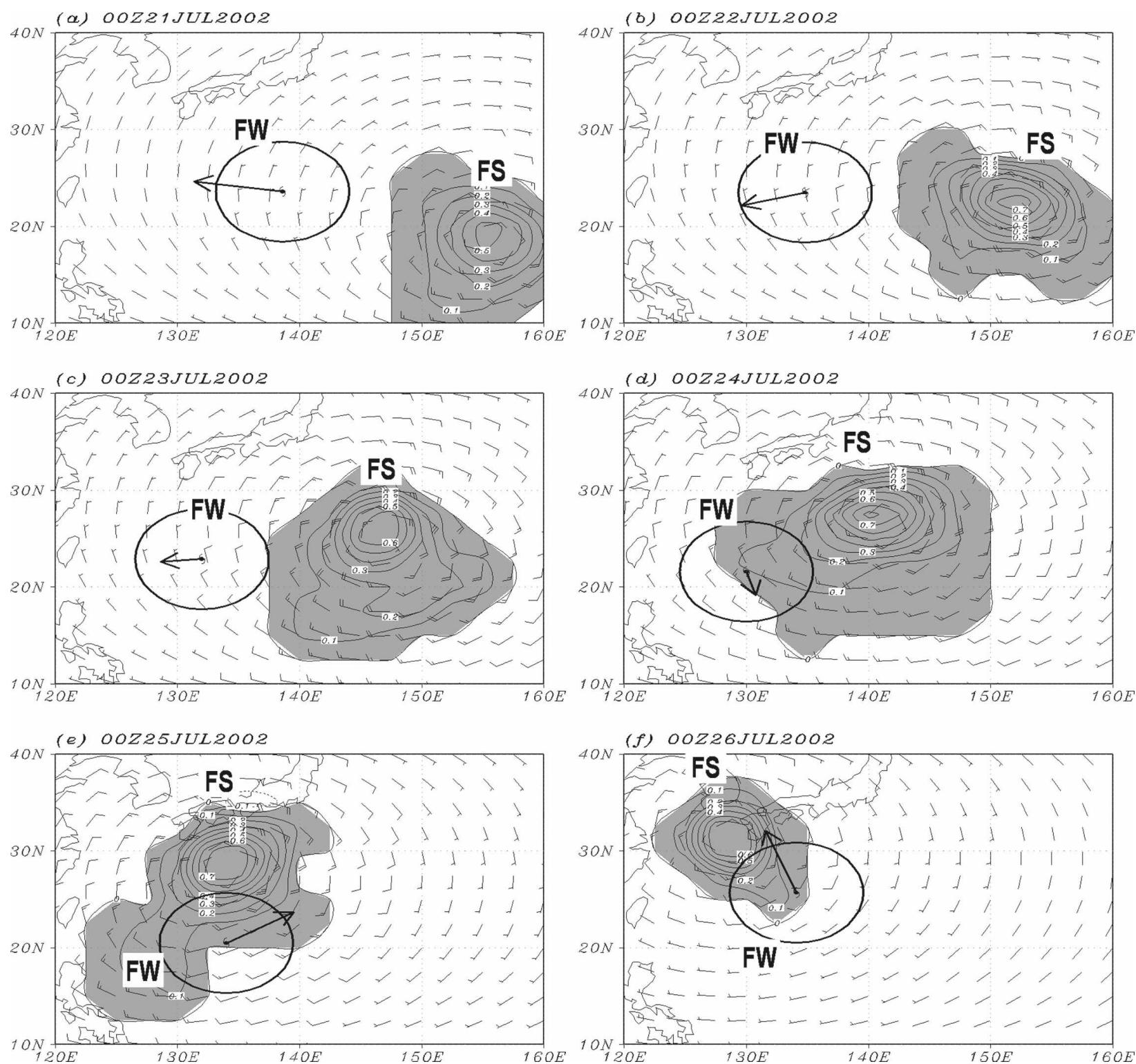

FIG. 5. As in Fig. 4 but for both the PV perturbation and the balanced 925-300-hPa deep-layer-mean steering wind [ $\mathbf{V}_{\text {SDLM }}\left(q_{\mathrm{FS}}^{\prime}\right)$; one full barb $=5 \mathrm{~m} \mathrm{~s}^{-1}$ ] associated with Fengshen, $q_{\mathrm{FS}}^{\prime}$, at $0000 \mathrm{UTC}$ (a) 21, (b) 22, (c) 23, (d) 24, (e) 25, and (f) $26 \mathrm{Jul} 2002$.

high; the monsoon trough that includes the PV perturbation of Fengshen); that is, $q^{\prime}=q_{\mathrm{FS}}^{\prime}+q_{\mathrm{noFS}}^{\prime}, q^{\prime}=$ $q_{\mathrm{SH}}^{\prime}+q_{\mathrm{noSH}}^{\prime}$, and $q^{\prime}=q_{\mathrm{MT}}^{\prime}+q_{\mathrm{noMT}}^{\prime}$.

A comparison between the wind bars of $\left[\mathbf{V}_{\text {SDLM }}\left(q^{\prime}\right)\right]$ and $\left[\mathbf{V}_{\mathrm{BT}}\right.$ (Fungwong)] in Fig. 6 shows that the balanced flow associated with the total PV perturbation $\left[\mathbf{V}_{\text {SDLM }}\left(q^{\prime}\right)\right]$ is, in general, consistent with the motion of the best track [ $\mathbf{V}_{\mathrm{BT}}$ (Fungwong)]. Both the best track and the perturbation steering flow of the subtropical high, [ $\left.\mathbf{V}_{\text {SDLM }}\left(q_{\mathrm{SH}}^{\prime}\right)\right]$, head westward from 0000 UTC 20 July to 0000 UTC 23 July, indicating that the main factor affecting Fungwong's motion is the subtropical high.
In addition, Fungwong's cyclonic turning movement $\left[\mathbf{V}_{\mathrm{BT}}\right.$ (Fungwong)] is rather consistent with the turning of the steering flow associated with Fengshen $\left[\mathbf{V}_{\text {SDLM }}\right.$ $\left.\left(q_{\mathrm{FS}}^{\prime}\right)\right]$ from 1200 UTC 23 July to 0000 UTC 25 July. Both vectors turn while pointing southwestward, southward, southeastward, and northeastward at 1200 UTC 23 July, 0000 UTC 24 July, 1200 UTC 24 July, and 0000 UTC 25 July, respectively. As the distance between Fengshen and Fungwong gradually increases after 0000 UTC 26 July, the subtropical high $\left(q_{\mathrm{SH}}^{\prime}\right)$ regains control over the motion of Fungwong.

In Fig. 6, the direction of the steering flow associated 


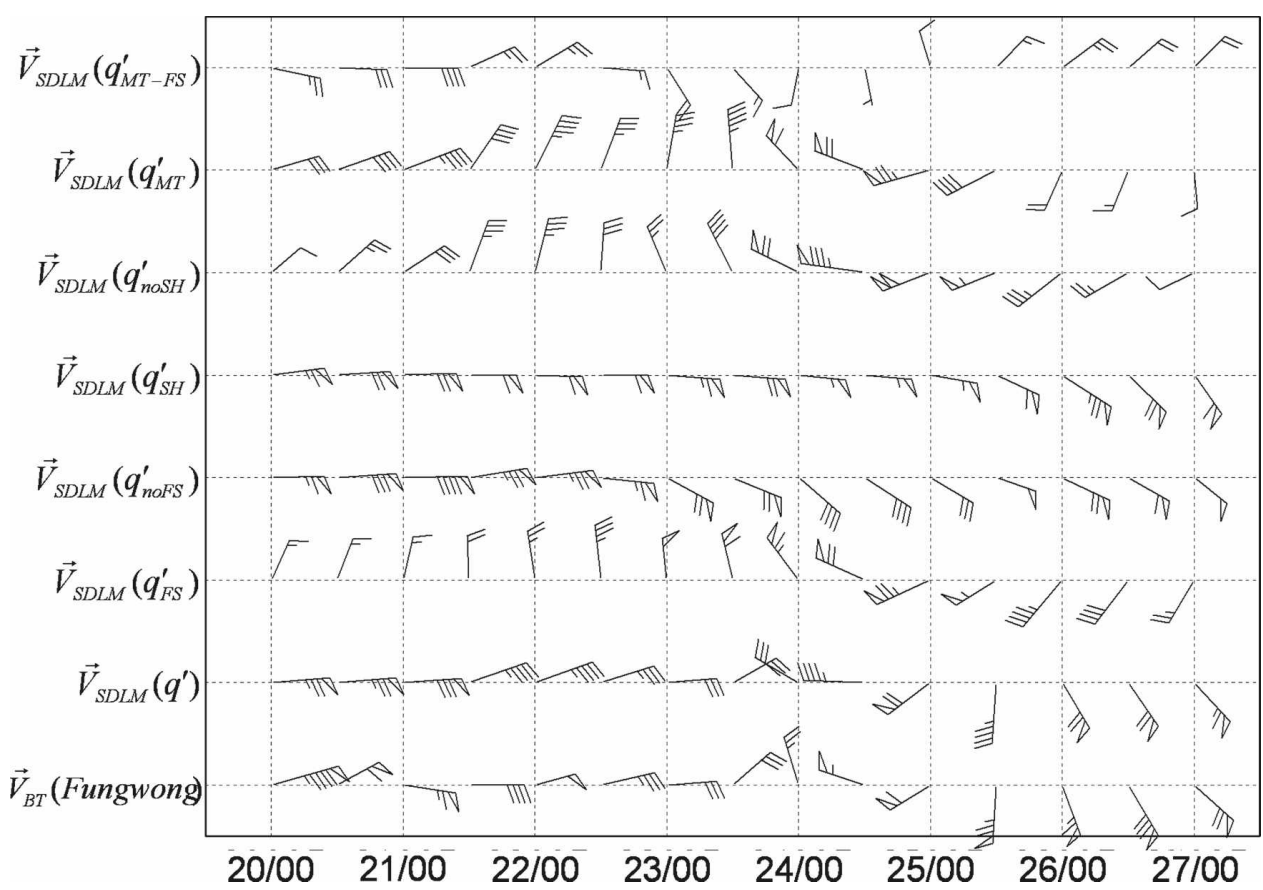

FIG. 6. Time series of Fungwong's movement ([ $\mathbf{V}_{\mathrm{BT}}$ (Fungwong) $\left.]\right)$ and the steering flow associated with the total PV perturbation $\left[\mathbf{V}_{\text {SDLM }}\left(q^{\prime}\right)\right]$, Fengshen $\left[\mathbf{V}_{\text {SDLM }}\left(q_{\mathrm{FS}}^{\prime}\right)\right]$, all perturbations but Fengshen $\left[\mathbf{V}_{\text {SDLM }}\right.$ $\left.\left(q_{\mathrm{noFS}}^{\prime}\right)\right]$, the subtropical high $\left[\mathbf{V}_{\mathrm{SDLM}}\left(q_{\mathrm{SH}}^{\prime}\right)\right]$, all perturbations but the subtropical high [ $\left.\mathbf{V}_{\mathrm{SDLM}}\left(q_{\mathrm{noSH}}^{\prime}\right)\right]$, the monsoon trough $\left[\mathbf{V}_{\mathrm{SDLM}}\left(q_{\mathrm{MT}}^{\prime}\right)\right]$, and all monsoon trough perturbations but Fengshen $\left[\mathbf{V}_{\text {SDLM }}\left(q_{\text {MT-FS }}^{\prime}\right)\right]$ from 0000 UTC 20 Jul to 0000 UTC $27 \mathrm{Jul} 2002$. One full barb is $1 \mathrm{~m} \mathrm{~s}^{-1}$, and a triangle represents $5 \mathrm{~m} \mathrm{~s}^{-1}$.

with the monsoon trough $\left[\mathbf{V}_{\mathrm{SDLM}}\left(q_{\mathrm{MT}}^{\prime}\right)\right]$ also turns counterclockwise, a pattern similar to that associated with Fengshen $\left[\mathbf{V}_{\mathrm{SDLM}}\left(q_{\mathrm{FS}}^{\prime}\right)\right]$. Detailed comparison of the two vectors [i.e., difference of $\mathbf{V}_{\mathrm{SDLM}}\left(q_{\mathrm{MT}}^{\prime}\right)$ and $\mathbf{V}_{\text {SDLM }}\left(q_{\mathrm{FS}}^{\prime}\right)$ shown at the top row in Fig. 6] indicates that, relative to Fengshen, the large-scale monsoon trough provides more westward steering flow to Fungwong during the early stage (from 0000 UTC 20 July to 0000 UTC 23 July), slightly more northward flow during the middle stage (from 0000 UTC 23 July to 1200 UTC 25 July), and then more southwestward steering flow during the later stage (from 1200 UTC 25 July to 0000 UTC 27 July). Further discussion of the role of the monsoon trough is presented in section $5 \mathrm{c}$.

\section{2) Movement of Fengshen}

On the other hand, to evaluate the factors affecting the motion of Fengshen, the center of Fengshen is also chosen to construct the mean part of the PV diagnosis. The results (not shown here) indicate that Fengshen is mainly guided by the subtropical high, instead of Fungwong's circulation. The influence of Fungwong on Fengshen's motion is much weaker as compared with other perturbations before 1200 UTC 24 July. Never- theless, Fungwong exerts somewhat stronger steering flow over Fengshen from 1200 UTC 24 July to 0000 UTC 26 July.

Results of the steering flow analysis above demonstrate that during the period when the binary interaction occurs, the track of the weaker storm (Fungwong) is affected by the circulation associated with the stronger storm (Fengshen). Note that the large-scale environmental flow (mainly associated with the subtropical high) continues to steer Fengshen and Fungwong westward from 0000 UTC 20 July to 0000 UTC 26 July. Meanwhile, from 1200 UTC 23 July to 0000 UTC 25 July, Fungwong is clearly affected by the steering flow associated with Fengshen, while Fengshen is also under Fungwong's influence from 1200 UTC 24 July to 0000 UTC 26 July.

\section{c. AT analysis}

\section{1) Movement of Fungwong}

To quantify the influence of the steering flow with respect to each PV perturbation, as described in Eq. (4), the normalized component of the steering vector associated with each perturbation in the direction parallel to the steering vector, which is associated with all PV perturbations, is represented as AT. For example, 


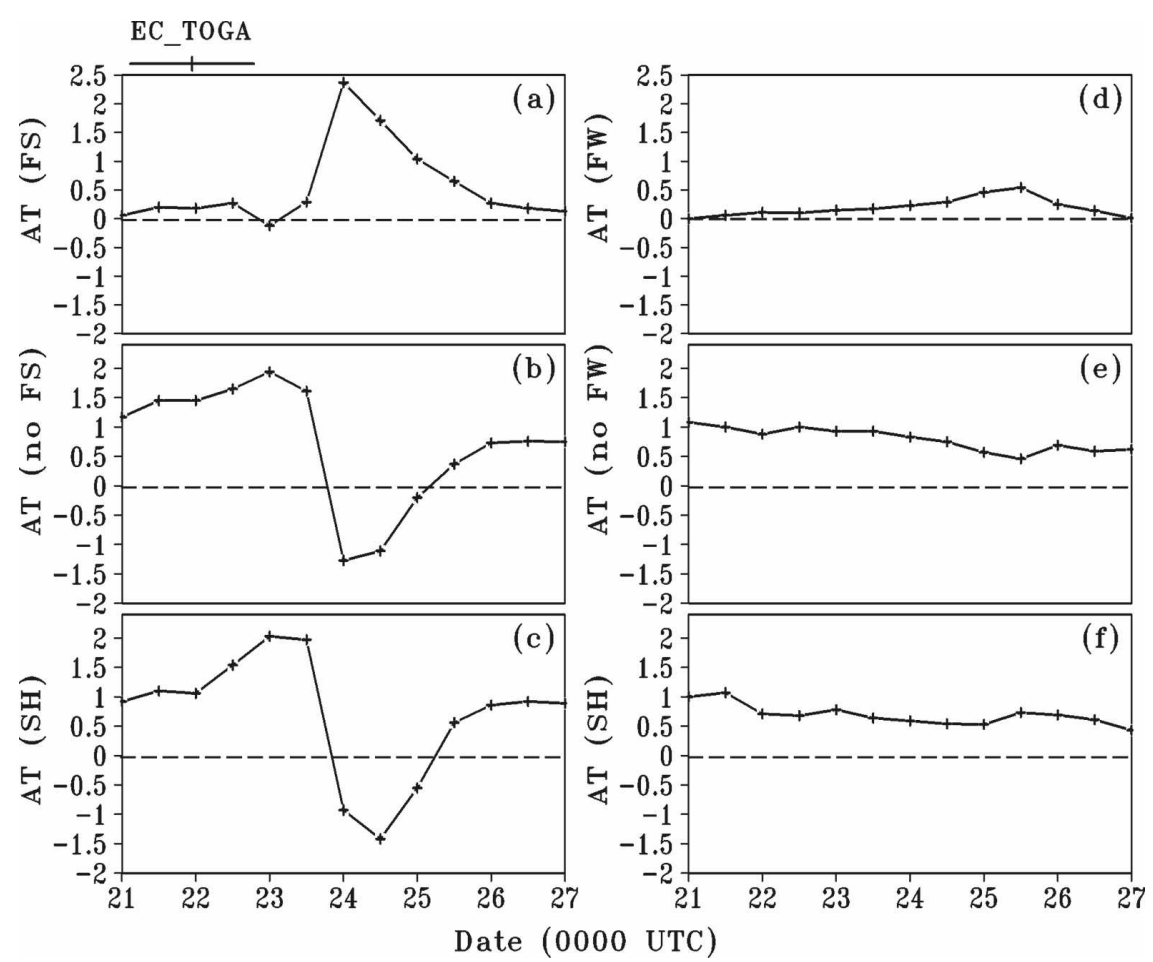

FIG. 7. Time series of AT associated with each perturbation from 0000 UTC 21 Jul to 0000 UTC 27 Jul. (a) AT(FS), (b) AT(no FS), and (c) AT(SH) with Fungwong are taken as the mean flow. (d) AT(FW), (e) AT(no FW), and (f) AT(SH) with Fengshen are taken as the mean flow.

$\operatorname{AT}\left(q_{\mathrm{FS}}^{\prime}\right)$ and $\operatorname{AT}\left(q_{\mathrm{SH}}^{\prime}\right)$ indicate the relative contributions to the motion of Fungwong with respect to the PV perturbation of Fengshen and the subtropical high, respectively. As shown in Fig. 7 , in the first 3 days, the value of $\operatorname{AT}\left(q_{\mathrm{FS}}^{\prime}\right)$ is maintained at about 0.1 ; however, at 0000 UTC 23 July, it starts to increase and then reaches its maximum of about 2.3, at 0000 UTC 24 July. The value of $\operatorname{AT}\left(q_{\mathrm{FS}}^{\prime}\right)$ remains higher than unity until 0000 UTC 25 July, indicating the important role Fengshen plays in steering Fungwong. Meanwhile, the value of $\operatorname{AT}\left(q_{\mathrm{SH}}^{\prime}\right)$ is kept higher than unity during the first 3 days and then drops to -1.5 from 0000 UTC 24 July to 0000 UTC 25 July. This implies that the subtropical high acts to impede Fungwong's movement from 24 to 25 July. As the results reveal in section 4b, Fungwong's movement is associated with the subtropical high from 21 to 23 July, while it is later strongly influenced by Fengshen from 24 to 25 July. Moreover, the steering flow associated with the subtropical high that decelerated Fungwong's movement in the later stage, from 24 to 25 July, is illustrated in Fig. 7c.

\section{2) Movement of Fengshen}

The analysis of AT with respect to the motion of Fengshen is shown in Figs. 7d, 7e, and 7f. The influence of Fungwong on Fengshen is too weak to be detected as compared with that of the subtropical high before 1200 UTC 24 July. Subsequently, $\operatorname{AT}\left(q_{\text {FW }}^{\prime}\right)$ increases to more than 0.3, indicating some influence from Fungwong on Fengshen's motion. Note that the value of $\operatorname{AT}\left(q_{\mathrm{SH}}^{\prime}\right)$ is always higher than 0.5 , implicating the persistent influence of the subtropical high on Fengshen's movement.

\section{d. The centroid-relative tracks}

The centroid-relative tracks based on geographic centers are often plotted to illustrate binary interaction in the literature. However, it has been noted that the geographic center may not really depict the actual interaction of two vortices with different strengths (Lander and Holland 1993; Carr et al. 1997; Carr and Elsberry 1998; and Wu et al. 2003). In this paper, the definition of the centroid from $\mathrm{Wu}$ et al. (2003) is used, that is, Eq. (5). The way that Fengshen and Fungwong mutually interact is illustrated in Fig. 8. In the beginning, Supertyphoon Fengshen moves slowly and is closer to the centroid while Fungwong moves rapidly and is farther away from the centroid-an example of the so-called one-way interaction. Nevertheless, after 


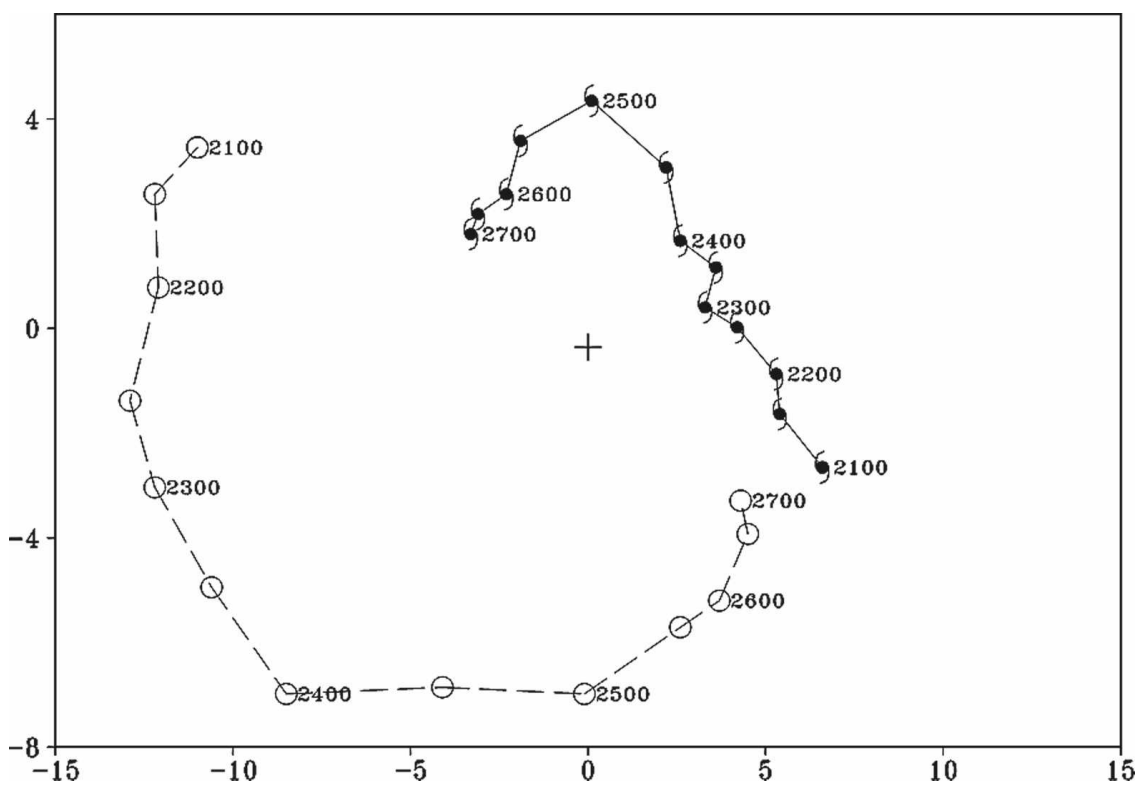

FIG. 8. Centroid-relative tracks of Fengshen (TC symbol) and Fungwong (circle) for every $12 \mathrm{~h}$ from 0000 UTC 21 Jul to 0000 UTC 27 Jul 2002.

1200 UTC 24 July, the mutual rotation is more apparent, indicating the presence of the two-way interaction.

Note that while Fungwong's looping motion is partly attributed to Fengshen's steering, it is also affected significantly by the westward steering flow associated with the subtropical high as shown by the above PV analysis. In all, the one-way and two-way interactions between Fungwong and Fengshen are well quantified by the above PV analysis, and the use of AT analysis and the centroid-relative tracks are also included at the same time.

\section{Results of the numerical simulation}

In this section, based on the MM5 model simulations, results of the sensitivities of the storm size, intensity, and the environmental flow to the binary interaction are presented.

\section{a. Control experiment}

Figure 9 presents the modeled track of CTRL, initiated at 0000 UTC 23 July, and the best track from the Joint Typhoon Warning Center (JTWC). In CTRL, Fungwong moves northwestward slowly for the first 12 simulated hours and then turns southward. At 1200 UTC 24 July, it moves southeastward while after 0000 UTC 25 July it speeds up toward the northeast and north-northeast. Except for the underestimation of the translation speed, the simulated Fengshen's track is rather consistent with the best track from the JTWC.
The intensity evolutions of Fengshen and Fungwong in CTRL and that from JMA are shown in Fig. 10. Fungwong's intensity is clearly underestimated by the numerical model, and the timing of the intensification is delayed by about $24 \mathrm{~h}$ as compared with the JMA analysis. On the other hand, the modeled intensity evolution of Fengshen is similar to the JMA analysis, except for the first $24 \mathrm{~h}$ of simulated time. Figure 10 also shows that the modeled 24-, 48-, and 72-h average forecast track errors are reasonably good and are approximately equals to the averaged official forecast track errors of the JTWC between 1998 and 2002 (from the JTWC Web site). In short, the entire simulation in CTRL reveals the binary interaction of the two storms and shows the reasonable track and intensity evolutions, except for underpredicting the intensity of Fungwong.

\section{b. Sensitivity study}

The modeled tracks of Fengshen and Fungwong in each sensitivity experiment are shown in Fig. 11. In B_FS (filled circle in Fig. 11), with Fengshen larger in size, Fungwong turns southward rapidly after the first 12-h simulation, indicating that Fungwong experiences Fengshen's impact slightly earlier than in CTRL. In S_FW (open square in Fig. 11), the modeled track of Fungwong in the first $36-\mathrm{h}$ simulation is almost consistent with that in FW (open circle), implying that with strengthened intensity Fungwong is not strongly influenced by Fengshen during this period. In W_FS (solid 


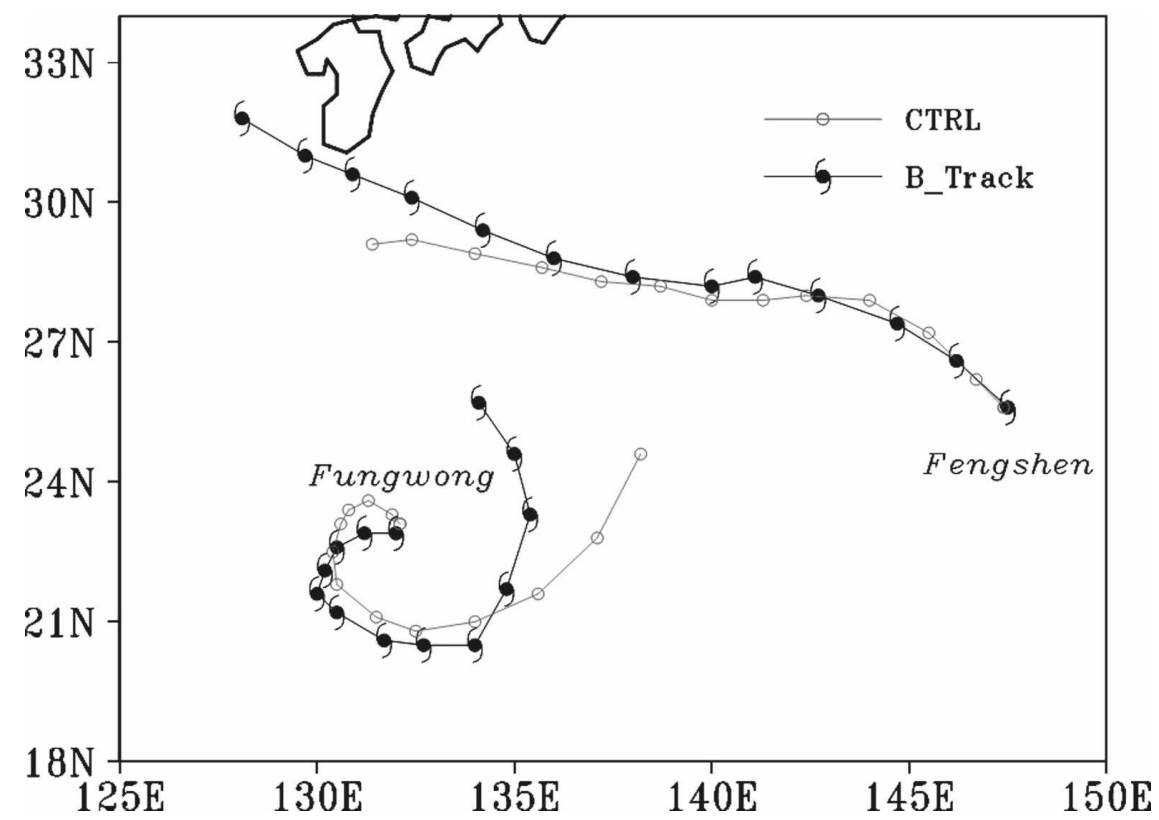

FIG. 9. JTWC's best tracks and the model tracks of Fengshen and Fungwong from 0000 UTC $23 \mathrm{Jul}$ to 0000 UTC $25 \mathrm{Jul}$.

square in Fig. 11), because the steering flow on Fungwong associated with Fengshen decreases as its intensity decreases, Fungwong's cyclonic looping lags behind the looping motion in CTRL by $12 \mathrm{~h}$. In FW (open circle), Fungwong still curves cyclonically without the presence of Fengshen, implying the potential influence of the large-scale MT system over the western North Pacific.

In contrast to the case of Fungwong, Fengshen's own simulated track is not qualitatively affected when its

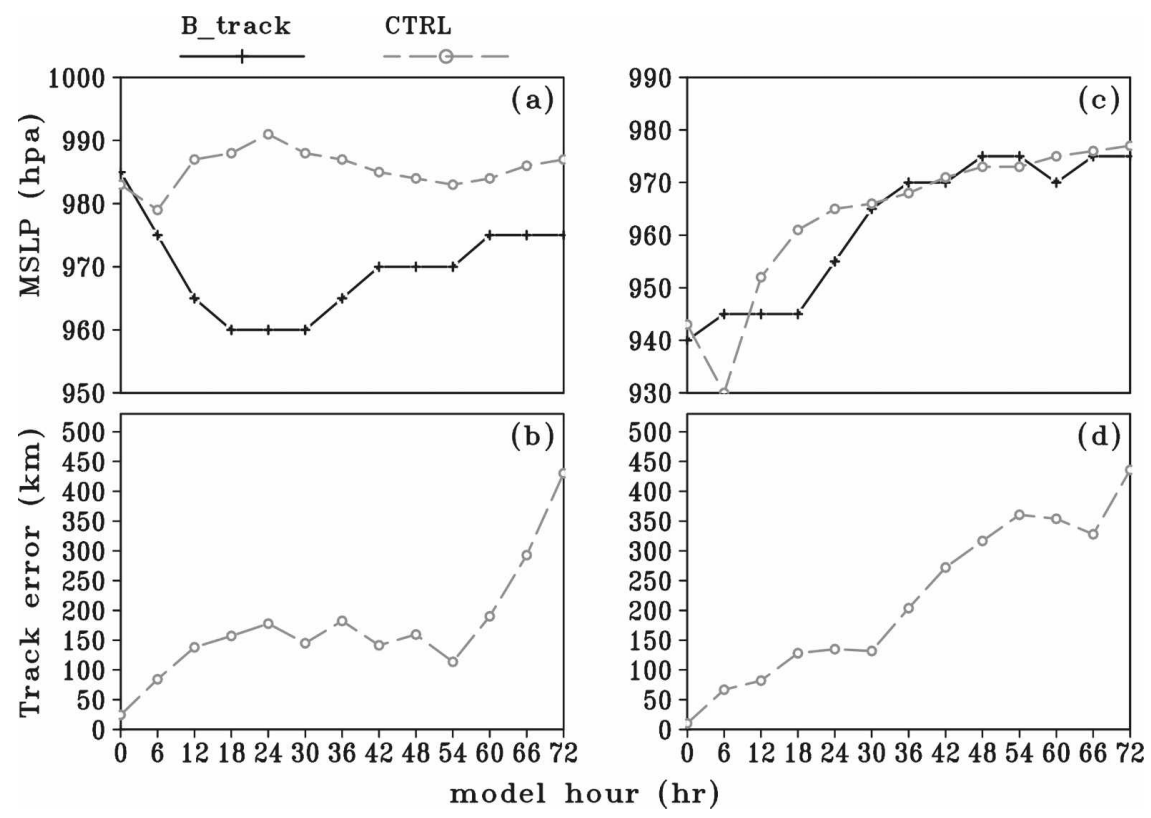

FIG. 10. Time series of the minimum central sea level pressure (hPa) from both JMA reports and CTRL of (a) Fungwong and (c) Fengshen, with the modeled track error (km) (verified against with the JTWC's best track) of (b) Fungwong and (d) Fengshen for every $6 \mathrm{~h}$ from 0000 UTC 23 Jul to 0000 UTC 26 Jul 2002. 


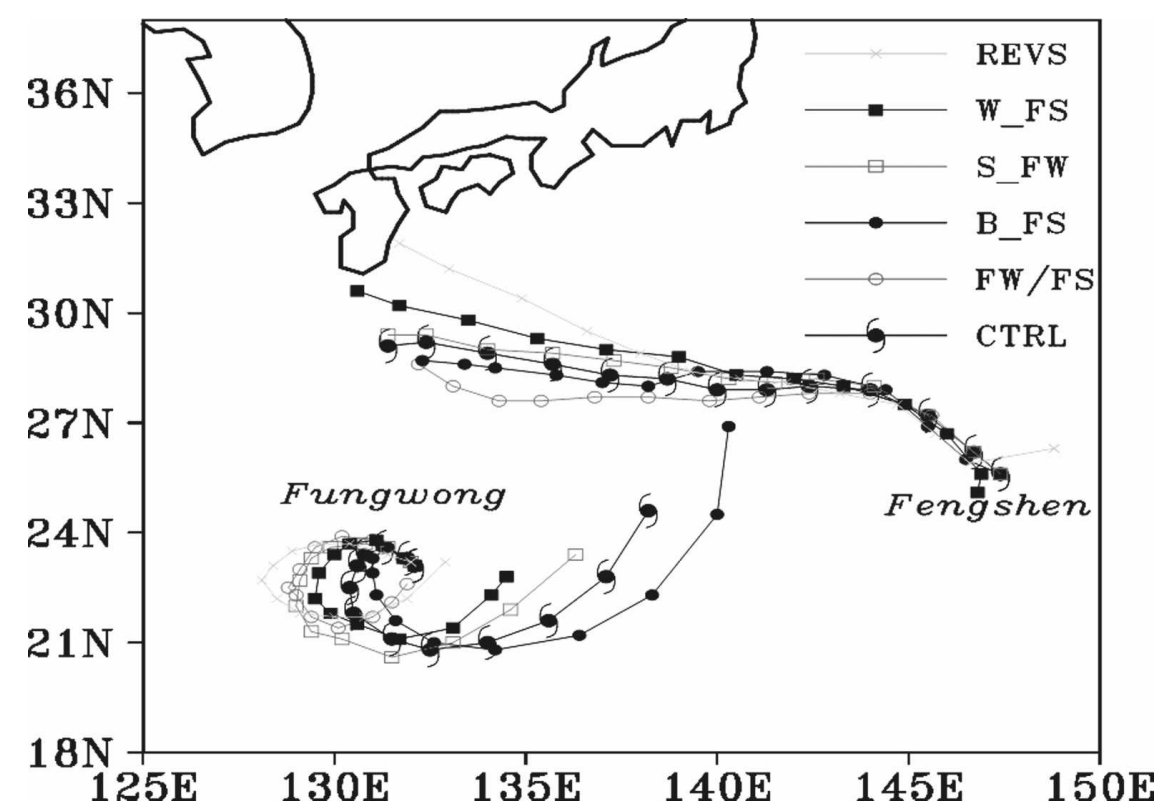

FIG. 11. Model tracks of all sensitivity experiments at 6-h interval from 0000 UTC 23 Jul to 0000 UTC 26 Jul 2002.

intensity or its radius is changed (in W_FS and B_FS). In W_FS, Fengshen moves faster than its analog in CTRL, which reveals that with the same environmental steering flow, the weaker storm moves faster than the stronger one. In FS (open circle in Fig. 11), Fengshen's forecast track is similar to that in CTRL initially, but moves due westward to the south of the tracks from the other experiment at $18 \mathrm{~h}$, that is, 1800 UTC 23 July.

The binary interaction process is sensitive to the initial conditions, especially in weaker storms (Fungwong, in this case). Figure 12b (Fig. 12d) shows differences in the tracks of Fungwong (Fengshen) between each sensitivity experiment, and the CTRL. The differences in Fungwong increases linearly with time in all experiments, while those in Fengshen remain less than $150 \mathrm{~km}$ during the 72-h simulations. The intensity evolutions of Fungwong and Fengshen in all experiments are displayed in Figs. 12a and 12c. In FW, B_FS, and W_FS, Fungwong's intensity in the first $36-\mathrm{h}$ simulation is consistent with that in CTRL, weakening in the beginning and restrengthening later on. After that, they bifurcate owing to their own binary interaction processes. In FW, Fungwong weakens slightly, and then strengthens again, whereas in B_FS, it continues to strengthen until $42 \mathrm{~h}$, and then weakens steadily. In W_FS, it weakens with some oscillation.

It is notable that Fungwong weakens significantly in both S_FW and REVS in the first $12 \mathrm{~h}$ while the model is experiencing the initial dynamic adjustment. However, the huge difference between their intensities is attributed to the different initial vortex radii. This reveals that with the same initial intensity of $944 \mathrm{hPa}$, a bigger vortex is less sensitive to the change of the environmental flow; thus, a bigger vortex is more effective in maintaining its intensity.

Clearly, the evolutions of Fengshen's intensity in both FS and S_FW are similar to that in CTRL (Fig. 12c). This result implies that with the same vortex structure, Fengshen's motion is not affected by the presence or the intensity variation of Fungwong. In other words, with sufficiently strong intensity and a larger size, the vortex intensity is not sensitive to variations of the environmental flow. In B_FS, Fengshen's intensity is maintained at about $10 \mathrm{hPa}$ lower than that of CTRL except in the initial model adjustment period. Compared to other experiments, Fengshen's intensity varies more gently in REVS and W_FS.

\section{c. PV diagnosis analysis}

The piecewise PV inversion method used above is also adopted to elucidate the binary interaction in each sensitive experiment. Based on the previous results, the steering flow associated with the total perturbation $\left[\mathbf{V}_{\text {SDLM }}\left(q^{\prime}\right)\right]$ represents well the storm motion. Figure 13 shows the total perturbation steering flow in CTRL, along with the difference between CTRL and other experiments, when the center of Fungwong is used to calculate the azimuthal average flow, as in Eq. (1). The total perturbation steering flows $\left[\mathbf{V}_{\text {SDLM }}\left(q^{\prime}\right)\right]$ associated with FW and CTRL are similar initially, thus lead- 


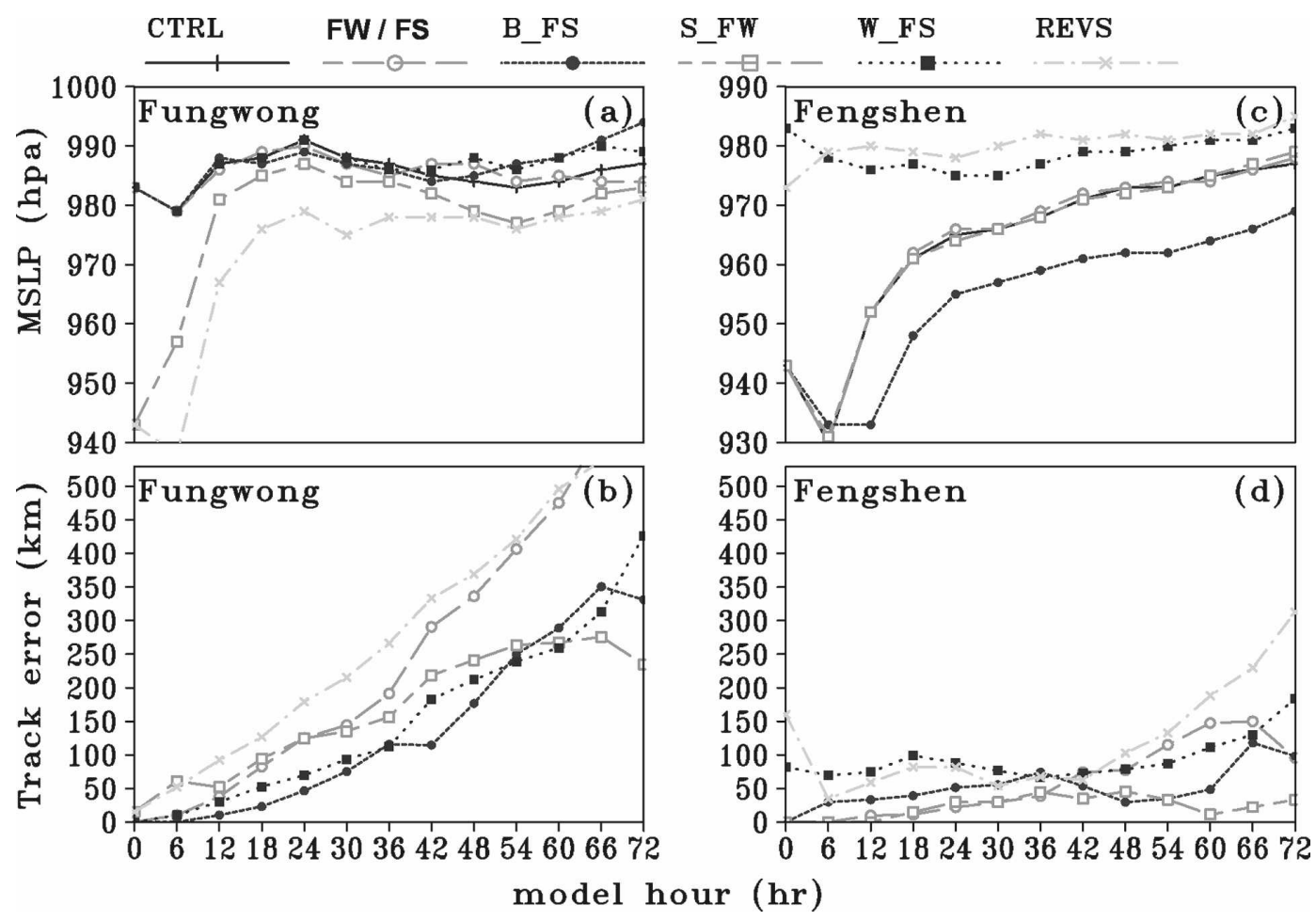

FIG. 12. Time series of the model's minimum central sea level pressure $(\mathrm{hPa})$ and the track difference relative to CTRL (km) from each sensitivity experiment from 0000 UTC 23 Jul to 0000 UTC 26 Jul 2002.

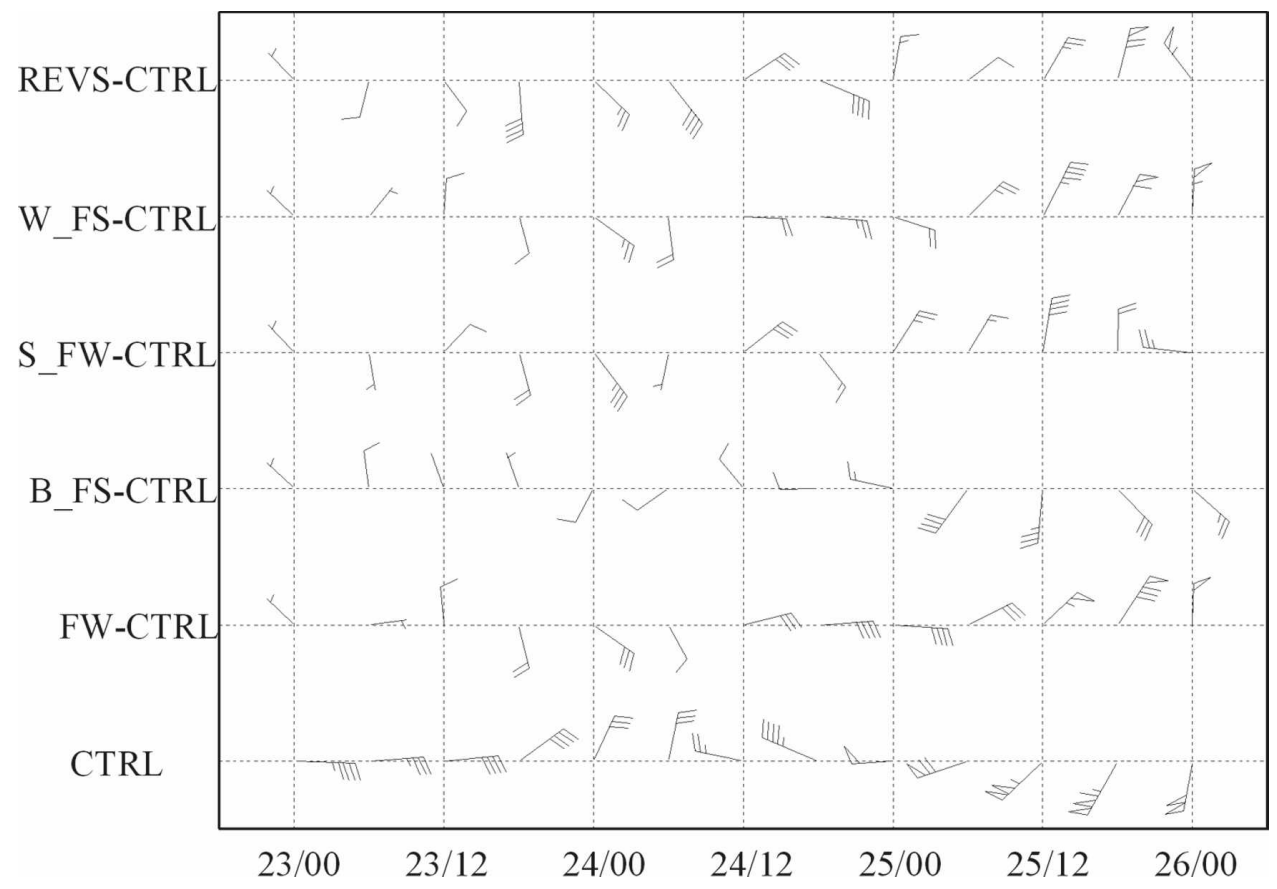

FIG. 13. Time series (from 0000 UTC 23 Jul to 0000 UTC 26 Jul 2002) of the steering flow associated with the total PV perturbation of CTRL while taking Fungwong as the mean flow. FW-CTRL indicates the differences in the steering flows associated with the total PV perturbations of FW and CTRL, respectively, and for B_FS-CTRL, S_FW-CTRL, W_FS-CTRL, and REVS-CTRL. One full barb is 1 $\mathrm{m} \mathrm{s}^{-1}$, and a triangle represents $5 \mathrm{~m} \mathrm{~s}^{-1}$. 


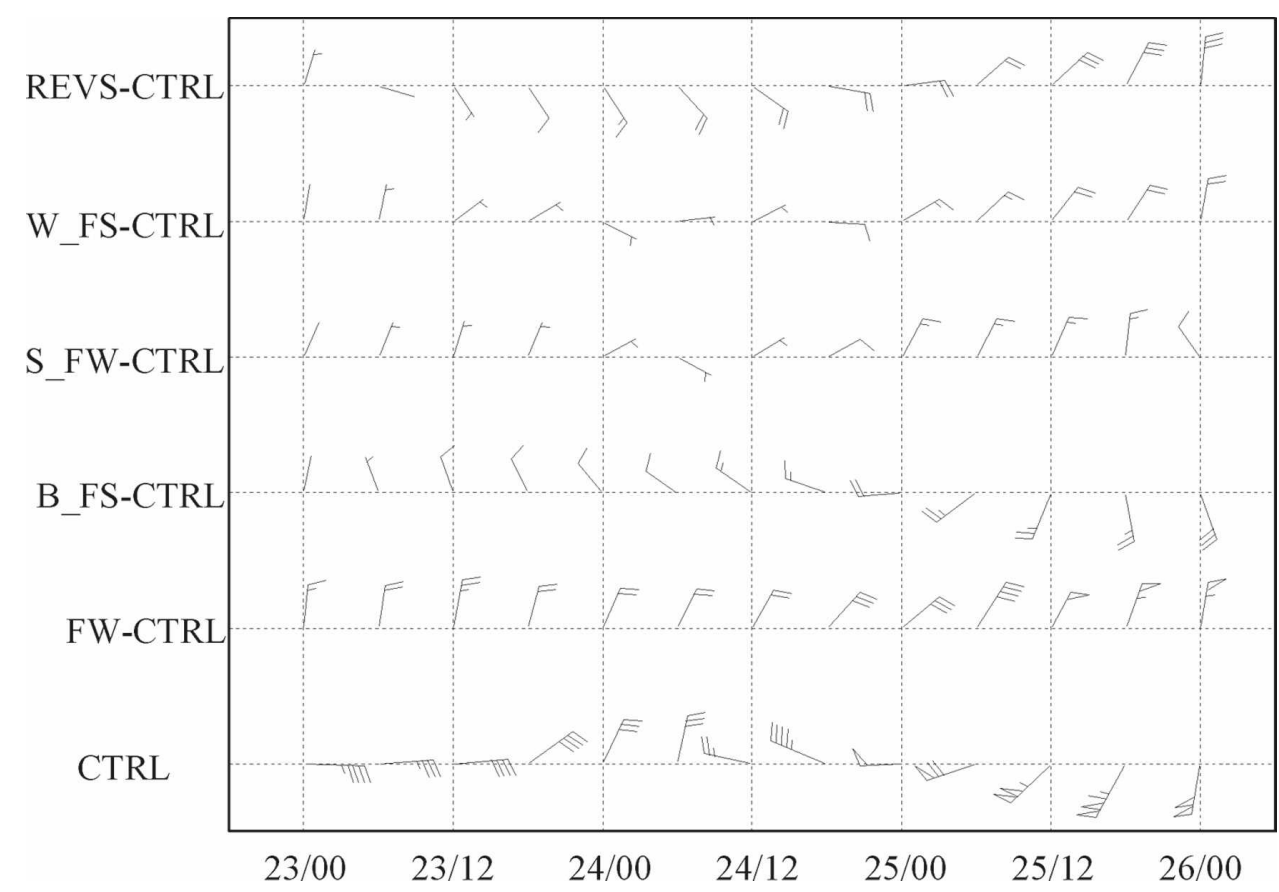

FIG. 14. As in Fig. 13 but for the perturbation associated with Fengshen.

ing to very small vectors in FW-CTRL (the difference in the steering flows associated with the total PV perturbations of FW and CTRL, respectively) in Fig. 13. In $\mathrm{FW}$, the steering flow is about $3-8 \mathrm{~m} \mathrm{~s}^{-1}$ weaker than that in CTRL after 1200 UTC 24 July, implying that Fungwong moves much slower in FW at a later simulated stage due to the lack of influence from Fengshen. Experiment B_FS-CTRL shows that the bigger Fengshen becomes, the more southward steering flow it would provide, thus making Fungwong turn southward earlier. Figure 13 also shows that Fungwong moves slower in S_FW, W_FS, and REVS.

A comparison of the steering flow of Fengshen $\left[\mathbf{V}_{\text {SDLM }}\left(q_{\mathrm{FS}}^{\prime}\right)\right]$ in CTRL, with those in B_FS, S_FW, W_FS, and REVS is displayed in Fig. 14. Note that there is no Fengshen vortex in FW, the comparison has been done on the steering flow associated with monsoon trough, $\left[\mathbf{V}_{\mathrm{SDLM}}\left(q_{\mathrm{MT}}^{\prime}\right)\right]$. B_FS-CTRL implies that $\left[\mathbf{V}_{\text {SDLM }}\left(q_{\mathrm{FS}}^{\prime}\right)\right]$ in B_FS is stronger than that in CTRL and their difference increases with time. On the other hand, in both S_FW and W_FS, [ $\left.\mathbf{V}_{\mathrm{SDLM}}\left(q_{\mathrm{FS}}^{\prime}\right)\right]$ is similar to that in CTRL before 0000 UTC 25 July, and becomes weaker later on. However, the reasons leading to the weaker steering flow in S_FW and W_FS are quite different. In S_FW, the magnitude of $\left[\mathbf{V}_{\text {SDLM }}\left(q_{\mathrm{FS}}^{\prime}\right)\right]$ dwindles because the stronger Fungwong with deeper vertical extent experiences less influence from Fengshen. In W_FS, the steering flow weakens since the weaker Fengshen offers weaker steering flow. FW-
CTRL shows that the monsoon trough exerts its influence on Fungwong with more southward and southeastward steering flows of about $2-5 \mathrm{~m} \mathrm{~s}^{-1}$ during most of the time period. Furthermore, as indicated from the steering flows associated with the $\mathrm{SH}$ and MT perturbations in FW (Fig. 15), Fungwong's initial southward deflection is likely attributed to the presence of the monsoon trough, while the counterclockwise looping may result from the combined influence of the monsoon trough and other synoptic systems, such as the subtropical high.

\section{d. Quantification of binary interaction}

The quantitative analyses done in section $4 \mathrm{c}$ are also conducted here to examine the characteristics of binary interaction in those numerical experiments. Figures 16a and $16 \mathrm{~b}$ show $\operatorname{AT}\left(q_{\mathrm{FS}}^{\prime}\right)$ and $\operatorname{AT}\left(q_{\mathrm{SH}}^{\prime}\right)$, respectively, when Fungwong is chosen as the mean flow, while Figs. $16 \mathrm{c}$ and $16 \mathrm{~d}$ display $\operatorname{AT}\left(q_{\mathrm{FW}}^{\prime}\right)$ and $\operatorname{AT}\left(q_{\mathrm{SH}}^{\prime}\right)$ when Fengshen is chosen as the mean flow. Note that in FW and $\mathrm{FS}$, both $\operatorname{AT}\left(q_{\mathrm{FS}}^{\prime}\right)$ and $\operatorname{AT}\left(q_{\mathrm{FW}}^{\prime}\right)$ represent the steering flow associated with monsoon trough. In CTRL, $\operatorname{AT}\left(q_{\mathrm{FS}}^{\prime}\right)$ is negative in the first 18 simulating hours, then gradually increases to unity, and finally decreases to about 0.5 . Meanwhile, $\operatorname{AT}\left(q_{\mathrm{FW}}^{\prime}\right)$ remains at 0.2 in the beginning, and then increases to 0.5 at $36 \mathrm{~h}$. Therefore, the binary interaction in CTRL is a one-way interaction before 1800 UTC $23 \mathrm{July}$, and then it turns into a mu- 


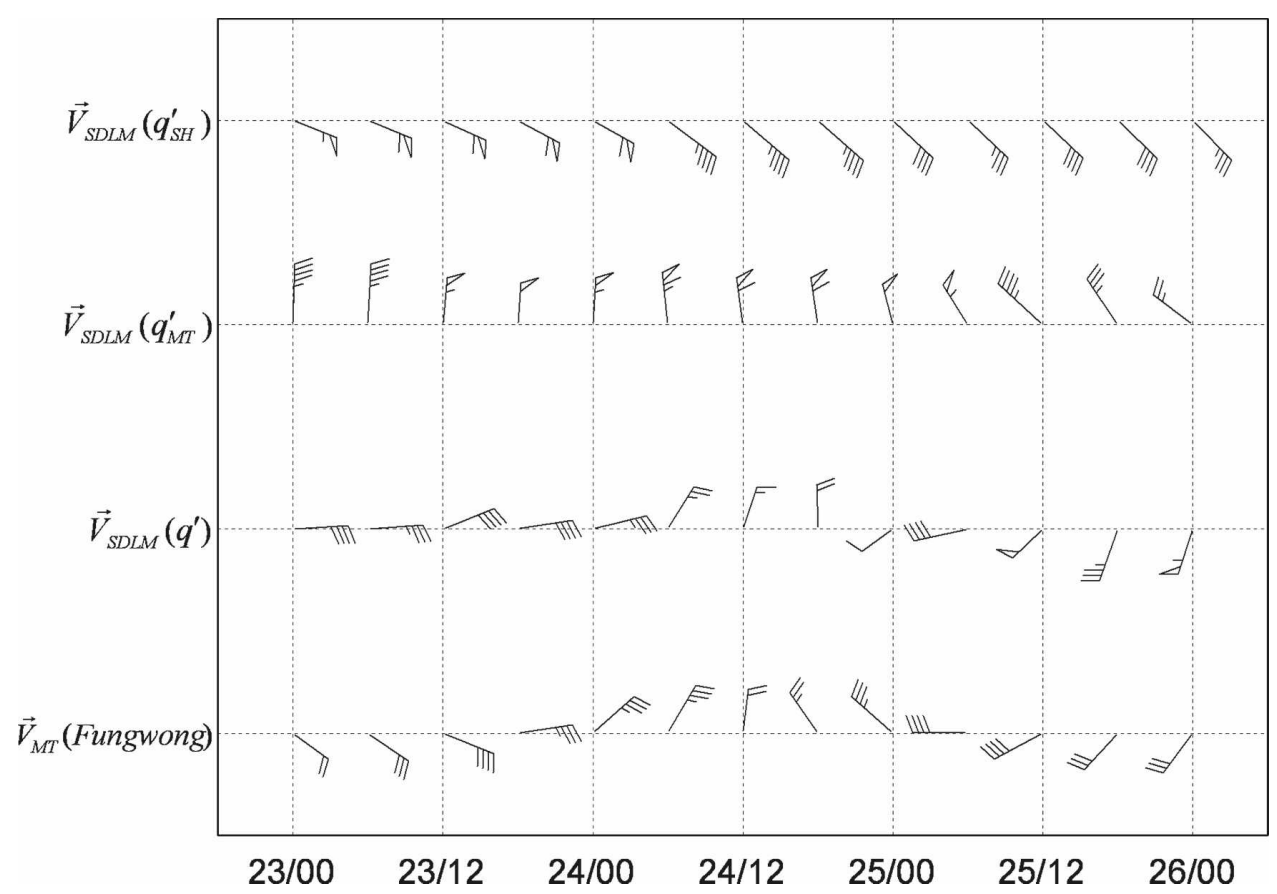

FIG. 15. As in Fig. 6 but for the PV analysis of the model results from FW.

tual interaction at 1200 UTC 24 July, consistent with the synoptic analysis in section $4 \mathrm{~d}$.

In all of the sensitivity experiments, $\operatorname{AT}\left(q_{\mathrm{FS}}^{\prime}\right)$ tends to increase sharply to the maximum value some time during the 30-36-h model integration (Fig. 16a). Figures $16 \mathrm{a}$ and $16 \mathrm{~b}$ imply that in all of our numerical experiments the motion of Fungwong is mainly affected by the subtropical high at the early integration stage, and is then gradually controlled by Fengshen later on. However, when Fungwong turns southward in the later stage, the subtropical high plays an obstructive role in preventing Fungwong from moving eastward.

In Fig. 16c, $\operatorname{AT}\left(q_{\mathrm{FW}}^{\prime}\right)$ shows the influence of Fungwong on Fengshen's motion. It remains at a value between 0.2 and 0.45 in all of our experiments. The contribution of the subtropical high to Fengshen, $\operatorname{AT}\left(q_{\mathrm{SH}}^{\prime}\right)$, is higher than 0.5 all the time, thus indicating that the steering flow associated with the subtropical high is the main factor that controls Fengshen's motion in the numerical experiments (Fig. 16d). These results are all consistent with the above analyses in section 4 .

\section{e. The centroid-relative tracks}

One of the most important issues in binary interaction is clarifying the type of binary interaction. The centroid-relative track used in each sensitivity experiment discussed in section $5 \mathrm{~b}$ is displayed in Fig. 17. In CTRL, the centroid-relative track resembles that from the analysis data (i.e., Fig. 8), showing the one-way interaction in the earlier period and the two-way interaction at the later stage. With a larger Fengshen in B_FS, the binary interaction is purely a one-way interaction. In other words, the influence of Fengshen on Fungwong is huge while that of Fungwong on Fengshen is very much limited (Fig. 17b). In S_FW (Fig. 17c), the centroid-relative track and the type of binary interaction are similar to that in CTRL. In W_FS and REVS (Figs. 17d and 17e), Fengshen moves close to the centroid slowly at first, but departs from the centroid rapidly later on. Contrary to Fengshen, Fungwong approaches the centroid gradually in the later stage while it is far away from the centroid in the beginning. The classification of the patterns in Figs. 17d and 17e are both one-way interactions (in different directions).

The presence of the different patterns of binary interactions, that is, the mutual interaction or the oneway interaction of different directions, is produced in the sensitivity experiments (Fig. 17). This highlights the potential impact of the uncertainty of the vortex structure on the simulation of binary interactions of TCs.

\section{Summary}

In 2002, Typhoon Fungwong ran its complete course within the lifetime of Supertyphoon Fengshen. Fung- 

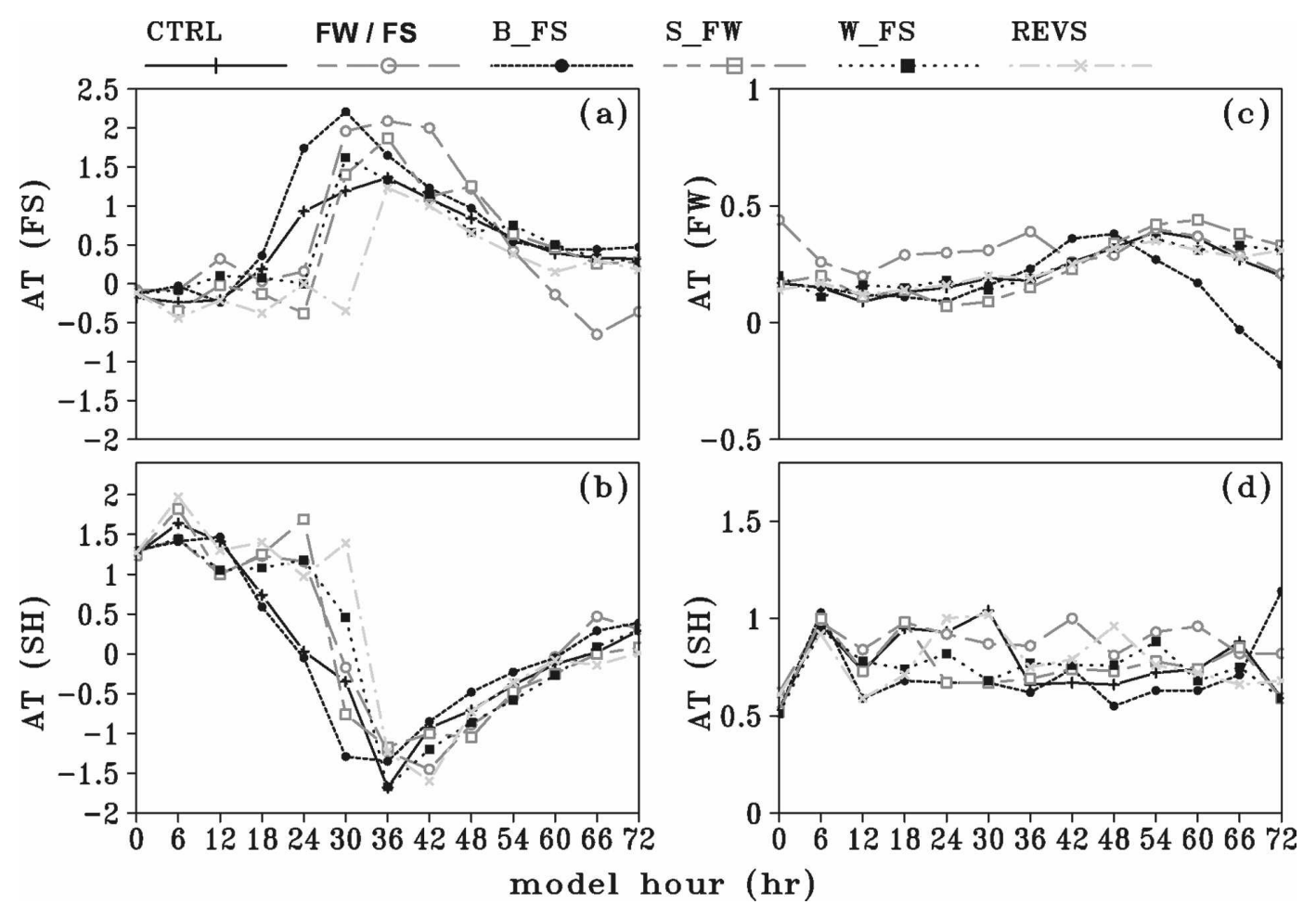

FIG. 16. Time series of AT of various PV perturbations from 0000 UTC 23 Jul to 0000 UTC 26 Jul in each experiment by taking (a), (b) Fungwong and (c), (d) Fengshen as the mean flow.

wong's track was controlled by Supertyphoon Fengshen and revealed cyclonic looping on 23 July 2002. The JTWC once classified this binary interaction as one-way interaction, that is, Fengshen affected Fungwong's motion whereas Fungwong had limited influence on Fengshen. This concept, however, is not totally correct. To further clarify the binary interaction between Fengshen and Fungwong, the PV diagnosis method as applied in $\mathrm{Wu}$ et al. (2003, 2004), as well as further numerical simulations, are adopted to study the sensitivity of this issue.

Results show that Fungwong's southward and northeastward movements are primarily induced by Fengshen from 1200 UTC 23 July to 0000 UTC 25 July, and at 1200 UTC 24, Fungwong begins to provide Fengshen with the westward steering flow. In other words, Fungwong and Fengshen interact with each other after 1200 UTC 24 July. Clearly, from 1200 UTC 23 July to 1200 UTC 24 July, the binary interaction is classified as oneway interaction, whereas from 1200 UTC 24 July to 0000 UTC 26 July, it can be considered to be two-way interaction. In addition to the binary interaction, the environmental flow associated with the subtropical high also induces the westward steering flow to Fungwong during the binary interaction process, and at some point it plays a role in decelerating the looping of Fungwong.
Based on a series of numerical simulations, the sensitivity of the vortex characteristics on the binary interaction is demonstrated. These sensitivity experiments are used not only to explore the factors controlling the interaction of binary vortices, but also to further examine the impact of the uncertainty of the initial vortex conditions on the interaction of the nearby TCs. The influence of one storm on the other is related to both storm intensities (represented by the minimum central sea level pressure of the vortex). The influence of Fengshen on the strengthened Fungwong is limited, and that of the steering flow associated with Fengshen on Fungwong weakens with the decrease in Fengshen's intensity. In addition, with the same environmental flow, weaker storms move faster than stronger ones, and vortices with sufficient intensity and size are less sensitive to variations in the environmental flow.

Simulation results exhibited in CTRL are similar to results based on the analysis data. However, different patterns of binary interactions are produced in the sensitivity experiments. For Fengshen with a larger vortex, the binary interaction is purely a one-way interaction. In other words, the influence of Fengshen on Fungwong is huge while that of Fungwong on Fengshen is rather limited. When Fungwong is strengthened, the binary interaction process is similar to that in CTRL, 
Centroid Tracks of CTRL (2002/07/23/00 26/00 Z)

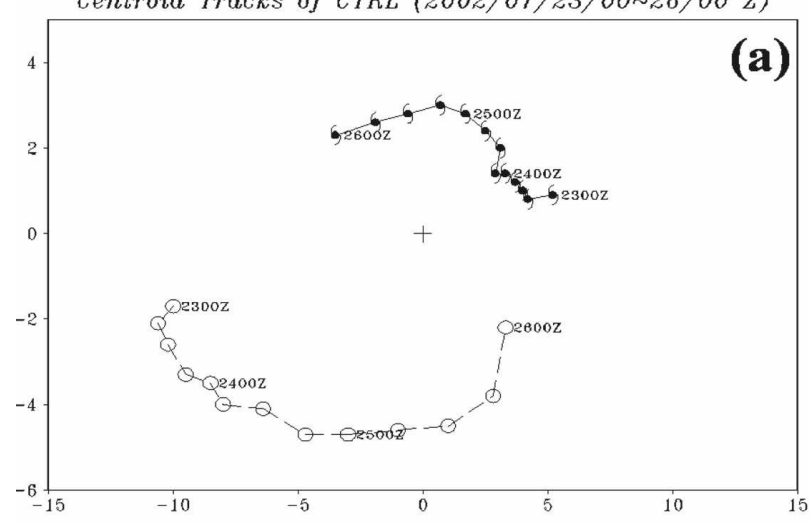

Centroid Tracks of S_FW (2002/07/23/00 26/00 Z)

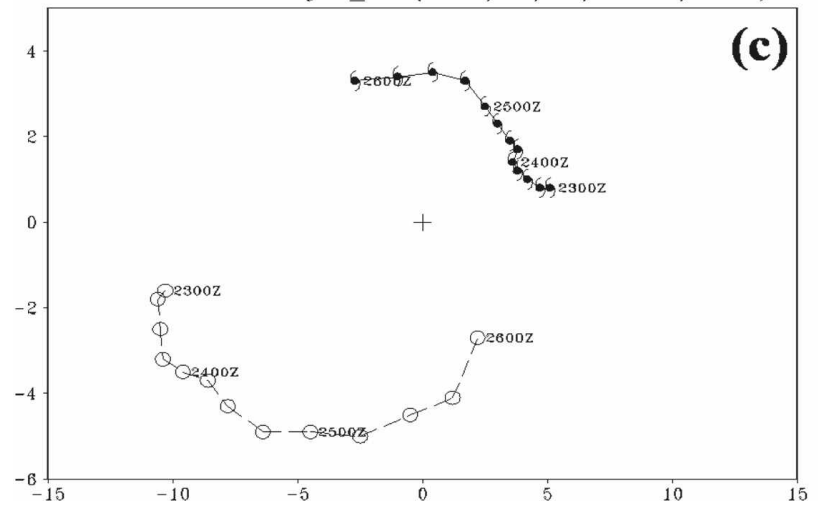

Centroid Tracks of REVS (2002/07/23/00 26/00 Z)

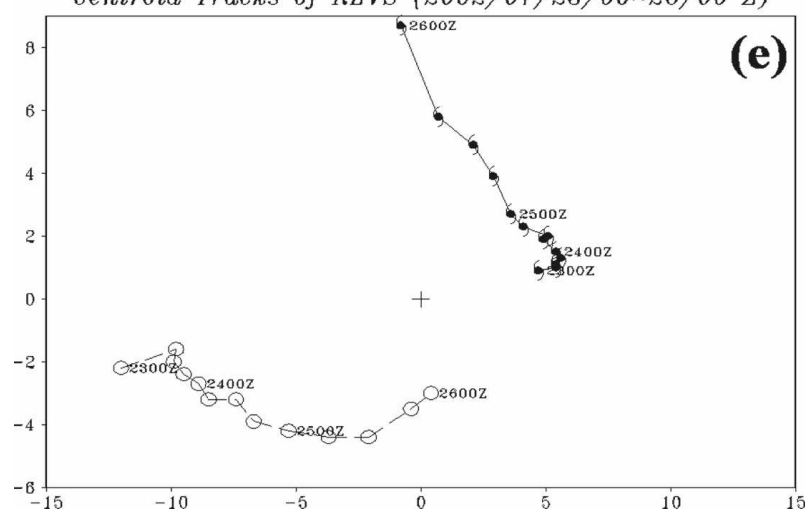

which begins with a one-way interaction and then turns into a two-way interaction. The classifications of the patterns in W_FS and REVS are both switched oneway interactions; that is, in the beginning Fengshen plays an active role in the binary interaction while Fungwong takes over the role later on. The potential impact of the uncertainty of the vortex structure on the simulation of binary interactions of TCs is thus indicated. In all, the result of this work further sheds light on the importance of the accurate representation of the initial vortices for a more profound understanding and improved prediction of nearby typhoons.
Centroid Tracks of BFS (2002/07/23/00 26/00 Z)

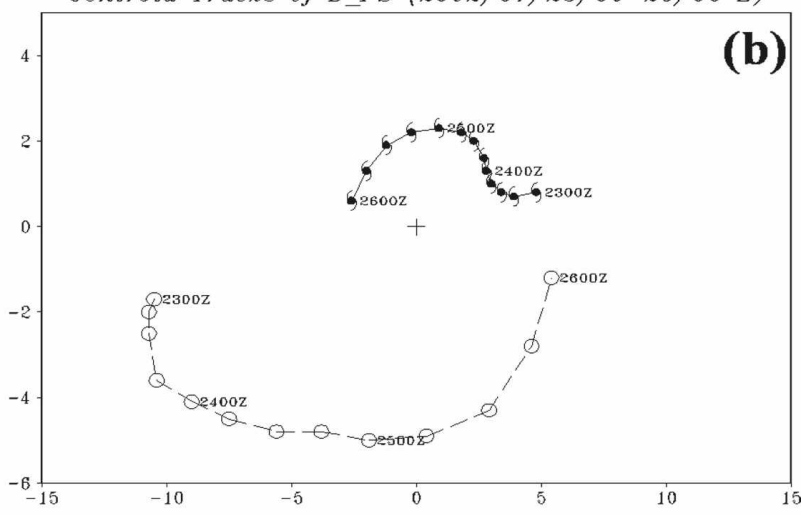

Centroid Tracks of W_FS (2002/07/23/00 26/00 Z)

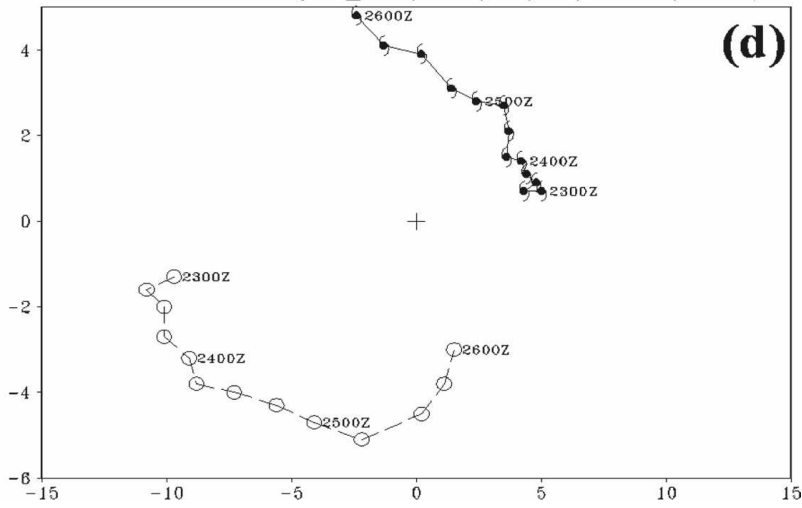

FIG. 17. Centroid-relative tracks of Fengshen (solid line) and Fungwong (dashed line) in each sensitivity experiment.

Finally, it should be pointed out that multiple nearby TCs and therefore binary interaction events, are often observed during the active monsoon season, in which a huge monsoon trough (or gyre) would generally exist. Under such circumstances, it is not easy to clearly separate the TC circulation from the circulation of the monsoon trough, making it difficult to isolate the binary interaction process. In this study, the numerical experiments and PV analyses clearly depict the signal of the binary interaction, while some additional impact from the monsoon trough appears to be nonnegligible. The role of the monsoon circulation in the binary interac- 
tion and in TC motion in general requires more careful investigation. This issue needs to be further addressed in future studies.

Acknowledgments. The work is supported through the National Science Council of Taiwan by Grant NSC95-2119-M-002-039-MY2, the Office of Naval Research by Grant N00014-05-1-0672, and the Central Weather Bureau by Grant MOTC-CWB-96-6M-02. The authors thank the anonymous reviewers for their very helpful comments.

\section{REFERENCES}

Anthes, R. A., and T. T. Warner, 1978: Development of hydrodynamic models suitable for air pollution and other mesometeorological studies. Mon. Wea. Rev., 106, 1045-1078.

— E.-Y. Hsie, and Y.-H. Kuo, 1987: Description of the Penn State/NCAR Mesoscale Model Version 4 (MM4). NCAR/ TN- 282+STR, Boulder, CO, 66 pp.

Brand, S., 1970: Interaction of binary tropical cyclones of the western North Pacific Ocean. J. Appl. Meteor., 9, 433-441.

Carr, L. E., III, and R. L. Elsberry, 1998: Objective diagnosis of binary tropical cyclone interactions for the western North Pacific basin. Mon. Wea. Rev., 126, 1734-1740.

— dence for alternate modes of track-altering binary tropical cyclone scenarios. Mon. Wea. Rev., 125, 2094-2111.

Chang, S. W., 1983: A numerical study of the interaction between two tropical cyclones. Mon. Wea. Rev., 111, 1806-1817.

_, 1984: Reply. Mon. Wea. Rev., 112, 1646-1647.

Davis, C. A., 1992: Piecewise potential vorticity inversion. J. Atmos. Sci., 49, 1397-1411.

DeMaria, M., and J. C. L. Chan, 1984: Comments on "A numerical study of the interactions between two tropical cyclones." Mon. Wea. Rev., 112, 1643-1645.

Dong, K., and C. J. Neumann, 1983: On the relative motion of binary tropical cyclones. Mon. Wea. Rev., 111, 945-953.

Dritschel, D. G., and D. W. Waugh, 1992: Quantification of the inelastic interaction of unequal vortices in two-dimensional vortex dynamics. Phys. Fluids, 4A, 1737-1744.

Fujiwhara, S., 1921: The natural tendency towards symmetry of motion and its application as a principle of motion. Quart. J. Roy. Meteor. Soc., 47, 287-293.

- 1923: On the growth and decay of vortical systems. Quart. J. Roy. Meteor. Soc., 49, 75-104.

- 1931: Short note on the behaviour of two vortices. Proc. Phys. Math. Soc. Japan, Ser. 3, 13, 106-110.

Holland, G. J., and G. S. Dietachmayer, 1993: On the interaction of tropical-cyclone-scale vortices. III: Continuous barotropic vortices. Quart. J. Roy. Meteor. Soc., 119, 1381-1398.

Lander, M. A., 1994: Description of a monsoon gyre and its effects on the tropical cyclones in the western North Pacific during August 1991. Wea. Forecasting, 9, 640-654.

_ 1996 : Specific tropical cyclone track types and unusual tropical cyclone motions associated with a reverse-oriented monsoon trough in the western North Pacific. Wea. Forecasting, 11, 170-186.

— cyclone-scale vortices. I: Observation. Quart. J. Roy. Meteor. Soc., 119, 1347-1361.

Prieto, R., B. D. McNoldy, S. R. Fulton, and W. H. Schubert, 2003: A classification of binary tropical-cyclone-like vortex interactions. Mon. Wea. Rev., 131, 2656-2666.

Ritchie, E. A., and G. J. Holland, 1993: On the interaction of tropical-cyclone-scale vortices. II: Discrete vortex patches. Quart. J. Roy. Meteor. Soc., 119, 1363-1379.

Shapiro, L. J., 1996: The motion of Hurricane Gloria: A potential vorticity diagnosis. Mon. Wea. Rev., 124, 2497-2508.

_, 1999: Potential vorticity asymmetries and tropical cyclone motion. Mon. Wea. Rev., 127, 124-131.

Wang, Y., and G. J. Holland, 1995: On the interaction of tropicalcyclone-scale vortices. IV: Baroclinic vortices. Quart. J. Roy. Meteor. Soc., 121, 95-126.

Wu, C.-C., and K. A. Emanuel, 1995a: Potential vorticity diagnostics of hurricane movement. Part I: A case study of Hurricane Bob (1991). Mon. Wea. Rev., 123, 69-92.

$\longrightarrow$, and $-1995 \mathrm{~b}$ : Potential vorticity diagnostics of hurricane movement. Part II: Tropical Storm Ana (1991) and Hurricane Andrew (1992). Mon. Wea. Rev., 123, 93-109.

— mechanisms of hurricane-environment interaction on hurricane movement from the potential vorticity perspective. $J$. Atmos. Sci., 53, 2264-2282.

— T.-H. Yen, Y.-H. Kuo, and W. Wang, 2002: Rainfall simulation associated with Typhoon Herb (1996) near Taiwan. Part I: The topographic effect. Wea. Forecasting, 17, 10011015.

— T.-S. Huang, W.-P. Huang, and K.-H. Chou, 2003: A new look at the binary interaction: Potential vorticity diagnosis of the unusual southward movement of Tropical Storm Bopha (2000) and its interaction with Supertyphoon Saomai (2000). Mon. Wea. Rev., 131, 1289-1300.

,,-- and K.-H. Chou, 2004: Potential vorticity diagnosis of the key factors affection the motion of Typhoon Sinlaku (2002). Mon. Wea. Rev., 132, 2084-2093.

Wu, L., and B. Wang, 2000: A potential vorticity tendency diagnostic approach for tropical cyclone motion. Mon. Wea. Rev., 128, 1899-1911.

- , and - 2001: Movement and vertical coupling of adiabatic baroclinic tropical cyclones. J. Atmos. Sci., 58, 1801-1814. 